\title{
Isatin-benzoazine molecular hybrids as potential antiproliferative agents: synthesis and in vitro pharmacological profiling
}

This article was published in the following Dove Press journal:

Drug Design, Development and Therapy

9 August 2017

Number of times this article has been viewed

\author{
Hatem A Abdel-Aziz' \\ Wagdy M Eldehna ${ }^{2}$ \\ Adam B Keeton ${ }^{3}$ \\ Gary A Piazza ${ }^{3}$ \\ Adnan A Kadi ${ }^{4}$ \\ Mohamed W Attwa ${ }^{4}$ \\ Ali S Abdelhameed ${ }^{4}$ \\ Mohamed I Attia ${ }^{4,5}$ \\ 'Department of Applied Organic \\ Chemistry, National Research \\ Centre, Giza, ${ }^{2}$ Department \\ of Pharmaceutical Chemistry, \\ Faculty of Pharmacy, Kafrelsheikh \\ University, Kafrelsheikh, Egypt; \\ ${ }^{3}$ Department of Oncologic Sciences \\ and Pharmacology, Drug Discovery \\ Research Center, Mitchell Cancer \\ Institute, University of South Alabama, \\ Mobile, AL, USA; ${ }^{4}$ Department of \\ Pharmaceutical Chemistry, College \\ of Pharmacy, King Saud University, \\ Riyadh, Saudi Arabia; ${ }^{5}$ Medicinal \\ and Pharmaceutical Chemistry \\ Department, Pharmaceutical and Drug \\ Industries Research Division, National \\ Research Centre, Giza, Egypt
}

Correspondence: Hatem A Abdel-Aziz Department of Applied Organic Chemistry, National Research Centre,

Dokki, Giza, PO Box 12622, Egypt

$\mathrm{Tel}+2122168624$

Fax +2 233370931

Email hatem_741@yahoo.com

Mohamed I Attia

Department of Pharmaceutical

Chemistry, College of Pharmacy,

King Saud University, PO Box 2457,

Riyadh I I45I, Saudi Arabia

Tel +966 | 4673764

Fax +966 | 4676220

Email mattia@ksu.edu.sa

\begin{abstract}
In continuation of our endeavor with respect to the development of potent and effective isatin-based anticancer agents, we adopted the molecular hybridization approach to design and synthesize four different sets of isatin-quinazoline $(\mathbf{6 a}-\mathbf{f}$ and $\mathbf{7 a}-\mathbf{e}) /$ phthalazine $(\mathbf{8 a}-\mathbf{f})$ /quinoxaline (9a-f) hybrids. The antiproliferative activity of the target hybrids was assessed towards HT-29 (colon), ZR-75 (breast) and A-549 (lung) human cancer cell lines. Hybrids 8b-d emerged as the most active antiproliferative congener in this study. Compound $\mathbf{8 c}$ induced apoptosis via increasing caspase $3 / 7$ activity by about 5 -fold in the A-549 human cancer cell line. In addition, it exhibited an increase in the G1 phase and a decrease in the $\mathrm{S}$ and G2/M phases in the cell cycle effect assay. Furthermore, it displayed an inhibitory concentration $50 \%$ value of $9.5 \mu \mathrm{M}$ against multidrug-resistant NCI-H69AR lung cancer cell line. The hybrid $\mathbf{8 c}$ was also subjected to in vitro metabolic investigations through its incubation with rat liver microsomes and analysis of the resulting metabolites with the aid of liquid chromatography-mass spectrometry.
\end{abstract}

Keywords: isatins, hybridization approach, antiproliferative, apoptosis

\section{Introduction}

In the current medical era, molecular hybridization approach has stood out as a valuable and important structural modification tool useful for the discovery and development of better therapies for diverse human diseases, mostly for cancer. ${ }^{1}$ The growing endeavors to discover hybrid drugs resulting from the combination of two or more haptophoric moieties of different bioactive substances have brought a new hope for the treatment of multifactorial disorders in recent years. Moreover, hybrid drugs can potentially overcome most of the pharmacokinetic drawbacks encountered by conventional anticancer drugs as well as provide combination therapies in a single multifunctional therapeutic agent at the target molecule conferring a more powerful, selective and safer drug compared to conventional classic treatments. ${ }^{2-4}$

Isatin ( $1 H$-indole-2,3-dione) is an endogenous compound found in many organisms, which was first isolated in 1988. ${ }^{5}$ As a privileged scaffold, isatin has emerged as an attractive and promising nucleus in the development of novel anticancer agents. ${ }^{6}$ Sunitinib (I) (Sutent ${ }^{\mathrm{TM}}$, Figure 1), granted the US Food and Drug Administration (FDA) approval in 2006, is an isatin-based orally active multi-targeted tyrosine kinase inhibitor used for the management of imatinib-resistant gastrointestinal stromal tumors and metastatic renal-cell carcinoma. ${ }^{7-9}$ By 2014, Nintedanib (II, Vargatef ${ }^{\mathrm{TM}}$, Figure 1), an orally available triple angiokinase inhibitor, was approved in the US for the treatment of idiopathic pulmonary fibrosis. One year later, the European Medicines submit your manuscript Dovepress f http:l/dx.doi.org/10.21 
<smiles>C#Cc1cccc(Nc2ncnc3cc(OCCOC)c(OCCOC)cc23)c1</smiles>

Sunitinib (I)

Nintedanib (II)

Erlotinib (III)<smiles>COc1cc2c(Nc3ccc(Br)cc3F)ncnc2cc1OCC1CCN(C)CC1</smiles>

Vandetanib (IV)<smiles>Clc1ccc(Nc2nnc(Cc3ccncc3)c3ccccc23)cc1</smiles>

Vatalanib (V)<smiles>O=C(c1cc(Cc2n[nH]c(=O)c3ccccc23)ccc1F)N1CCN(C(=O)C2CC2)CC1</smiles>

Olaparib (VI)

Figure I Chemical structures of clinically used anticancer agents.

Agency approved Nintedanib (II) as a second-line treatment in combination with docetaxel for non-small cell lung cancer of adenocarcinoma. ${ }^{10,11}$ Also, semaxanib and orantinib are other examples for isatin-based anticancer agents that are being used in clinical trials and possess multiple tyrosine kinase receptor inhibitory activities. ${ }^{12}$

Over the last decade, several studies suggested the significance of developing isatin-based hybrids as promising anticancer agents, ${ }^{13}$ among them, isatin-chromene VII,${ }^{14}$ isatin-pyridine VIIII, ${ }^{15}$ bis-isatin IX,${ }^{16,17}$ isatin-benzoxazole $\mathbf{X},{ }^{18}$ isatin-benzimidazole $\mathbf{X I},{ }^{19}$ isatin-benzothiazole, ${ }^{20}$ isatin-thiazolidine/thiazolidinone, ${ }^{21-25}$ isatin-4-piperazinylquinoline ${ }^{26}$ and isatin-pyrazoline ${ }^{27}$ hybrids were reported (Figure 2).

On the other hand, quinazolines constitute the cornerstone for a number of tyrosine kinase inhibitors such as the reversible EGFR-inhibitor. Erlotinib (III, Tarceva ${ }^{\mathrm{TM}}$,<smiles>[X][M]C1C(=O)Nc2ccc([R])cc2C1=NN=C1C(=O)Nc2ccc([R])cc21</smiles>

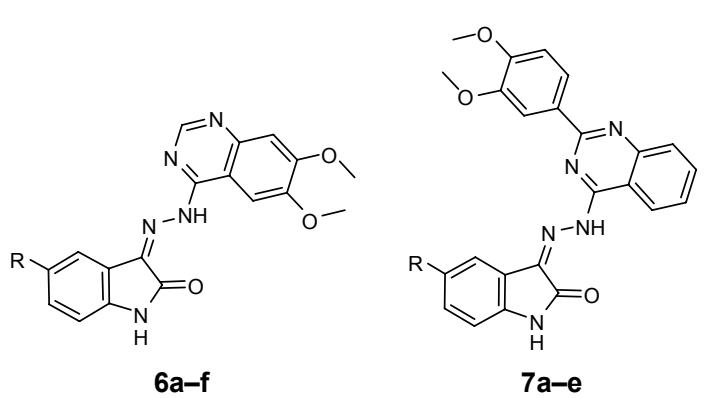

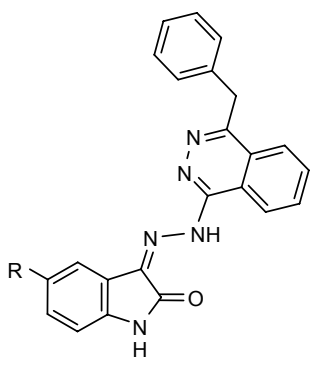

$8 a-f$<smiles>Cn1c(=O)c(N/N=C2/C(=O)Nc3ccc(Br)cc32)nc2ccccc21</smiles>

9a-f

Figure $\mathbf{2}$ Structures of some reported isatin-based hybrids with promising anticancer activity and structures of the target hybrids $\mathbf{6 a - f}, \mathbf{7 a - e , ~ 8 a - f}$ and $\mathbf{9 a - f}$. 
Figure 1), as well as the dual VEGFR-2-EGFR inhibitor Vandetanib (IV, Caprelsa ${ }^{\mathrm{TM}}$, Figure 1), is indicated for the treatment of symptomatic or progressive medullary thyroid cancer in patients with unresectable locally advanced or metastatic disease. ${ }^{28,29}$ Also, phthalazine is another attractive scaffold forming the backbone of certain promising antitumor lead candidates. Among them, Vatalanib (V, PTK787, Figure 1) is an orally active VEGFR-1 and VEGFR-2 inhibitor undergoing phase III clinical trials for the treatment of colorectal cancer. ${ }^{30,31}$ Olaparib (VI, Lynparza, Figure 1), the first approved phthalazine-based anticancer drug, is an oral small molecule poly (ADP-ribose) polymerase inhibitor that was approved in 2014 for the treatment of BRCA-mutated ovarian cancer. ${ }^{32}$ Recently, much attention has been paid to anticancer drug discovery based on the quinoxaline nucleus as an important heterocyclic one that exhibited interesting biological activities. ${ }^{33}$ It has been documented that several isatin-quinazoline and isatin-phthalazine hybrids displayed promising anticancer activities. ${ }^{34,35}$

In the light of the aforementioned findings and in continuation of our endeavor with respect to the development of potent and effective isatin-based anticancer agents, ${ }^{35,36}$ we adopted the molecular hybridization approach to design and synthesize four different sets of isatin-quinazolines $(\mathbf{6 a}-\mathbf{f}$ and $7 \mathbf{a}-\mathbf{e}) /$ phthalazines $(\mathbf{8 a}-\mathbf{f})$ /quinoxaline (9a-f) hybrids (Figure 2). All the synthesized hybrids (6a-f, 7a-e, 8a-f and $9 \mathbf{a}-\mathbf{f}$ ) were in vitro evaluated for their antiproliferative activity against three human cancer cell lines, namely human colon cancer HT-29 cell line, breast cancer ZR-75 cell line and lung cancer A-549 cell line. Moreover, the most active congeners were further assessed for their apoptosis induction potential using human cancer A-549 cell line, via evaluation of their effects on the expression of caspase $3 / 7$ as well as on the cell cycle progression, to obtain mechanistic insights into their anticancer activity. Furthermore, their antiproliferative activity against multidrug-resistant lung cancer NCI-H69AR cell line was evaluated. Finally, the most active candidates were subjected to in vitro metabolic investigations through their incubation with rat liver microsomes (RLMs) and analysis of the resulting metabolites with the aid of liquid chromatography-mass spectrometry (LC-MS).

\section{Materials and methods General}

Melting points of the synthesized compounds were measured with a Stuart melting point apparatus (Staffordshire, UK) and were uncorrected. Infrared (IR) spectra were recorded as $\mathrm{KBr}$ disks using FT-IR Spectrum BX apparatus (Perkin
Elmer, Shelton, CT, USA). Mass spectra were recorded using Agilent Quadrupole 6120 LC-MS with electrospray ionization (ESI) source (Agilent Technologies, Palo Alto, CA, USA). NMR spectra were recorded on a Bruker NMR spectrometer (Bruker Biospin, Billerica, MA, USA). ${ }^{1} \mathrm{H}$ spectra were run at 500 or $700 \mathrm{MHz}$, and ${ }^{13} \mathrm{C}$ spectra were run at 125 or $175 \mathrm{MHz}$ in deuterated dimethyl sulfoxide (DMSO- $d_{6}$ ). Chemical shifts are expressed in $\delta$ values (ppm) using the solvent peak as internal standard. All coupling constant $(J)$ values are given in hertz. The abbreviations used are as follows: s, singlet; d, doublet; m, multiplet. Elemental analyses were carried out at Microanalytical Centre, Cairo University, Egypt. High-resolution mass spectrometry (HRMS) measurements were performed on an LTQ-Orbitrap XL coupled to matrix-assisted laser desorption ionization (MALDI). Reaction courses and product mixtures were routinely monitored by thin layer chromatography on silica-gel precoated $\mathrm{F}_{254}$ Merck plates (Merck Millipore, Billerica, MA, USA). Unless otherwise noted, all solvents and reagents were commercially available and used without further purification. Compounds $\mathbf{1},{ }^{37} \mathbf{2},{ }^{35} 3{ }^{38}$ and $4^{39}$ were prepared according to the reported method. All cell lines were purchased from the American Type Culture Collection (ATCC) as follows: HT-29: (ATCC ${ }^{\circledR}$ HTB-38 ${ }^{\mathrm{TM}}$ ); ZR75: (ATCC ${ }^{\circledR}$ CRL-1500 $^{\mathrm{TM}}$ ); A549: $\left(\right.$ ATCC $^{\circledR}$ CRM-CCL-185 ${ }^{\mathrm{TM}}$ ); IEC-6: (ATCC ${ }^{\circledR}$ CRL-1592 ${ }^{\mathrm{TM}}$ ); 3T3Swiss albino $\left(\mathrm{ATCC}^{\circledR}\right.$ CCL-92 $\left.{ }^{\mathrm{TM}}\right)$; MCF 10A $\left(\mathrm{ATCC}^{\circledR}\right.$ CRL-10317 ${ }^{\mathrm{TM}}$ ); H69AR (ATCC ${ }^{\circledR}$ CRL-11351 ${ }^{\mathrm{TM}}$ ).

\section{Chemistry}

\section{General procedure for preparation of the target hybrids $\mathbf{6 a - f}, \mathbf{7 a - e , ~ 8 a - f}$ and $\mathbf{9 a - f}$}

The appropriate indoline-2,3-dione derivative $\mathbf{5 a}-\mathbf{f}(1 \mathrm{mmol})$ was added to a suspension of each hydrazinyl intermediate 1-4 (1 mmol) in ethanol (10 mL) and a catalytic amount of glacial acetic acid. The reaction mixture was refluxed for $1 \mathrm{~h}$. The precipitate formed was collected by filtration while hot, washed with hot ethanol, dried and re-crystallized from $\mathrm{DMF} / \mathrm{ethanol}$ to furnish the desired hybrids.

3-(2-(6,7-Dimethoxyquinazolin-4-yl)hydrazono) indolin-2-one (6a) - orange powder (yield 75\%), m.p. $297^{\circ} \mathrm{C}-299^{\circ} \mathrm{C}$; IR $\left(\mathrm{KBr}, v \mathrm{~cm}^{-1}\right): 3,411(\mathrm{NH})$ and 1,699 $(\mathrm{C}=\mathrm{O}) ;{ }^{1} \mathrm{H}$ NMR (DMSO- $\left.d_{6}\right) \delta$ ppm: $3.96\left(\mathrm{~s}, 3 \mathrm{H}, \mathrm{OCH}_{3}\right)$, $4.00\left(\mathrm{~s}, 3 \mathrm{H}, \mathrm{OCH}_{3}\right), 6.87-6.89(\mathrm{~m}, 2 \mathrm{H}, \mathrm{Ar}-\mathrm{H}), 7.17(\mathrm{t}, 1 \mathrm{H}$, Ar-H, $J=8.9 \mathrm{~Hz}), 7.21$ (s, 1H, Ar-H), 7.74 (s, 1H, Ar-H), 8.06 (s, 1H, Ar-H), 8.25 (d, 1H, Ar-H, J=8.8 Hz), 10.61 (s, 1H, $\mathrm{NH}), 12.36$ (s, 1H, NH); ${ }^{13} \mathrm{C}$ NMR (DMSO- $\left.d_{6}\right) \delta$ ppm: 55.8 $\left(\mathrm{OCH}_{3}\right), 56.58\left(\mathrm{OCH}_{3}\right), 104.2,108.6,111.1,111.2,112.9$, 114.4, 114.5, 117.4, 118.9, 139.6, 144.3, 149.6, 155.4, 157.2, 
158.5, $166.4(\mathrm{C}=\mathrm{O})$; MS (ESI) $m / z: 350.0[\mathrm{M}+\mathrm{H}]^{+}$; Anal. calcd. for $\mathrm{C}_{18} \mathrm{H}_{15} \mathrm{~N}_{5} \mathrm{O}_{3}$ (349.12): C, 61.89; H, 4.33; N, 20.05; found C, 62.13; H, 4.28; N, 20.11; HRMS (MALDI) calcd. for $\mathrm{C}_{18} \mathrm{H}_{15} \mathrm{~N}_{5} \mathrm{O}_{3}: 350.1253$, found: $350.1224[\mathrm{M}+\mathrm{H}]^{+}$.

3-(2-(6,7-Dimethoxyquinazolin-4-yl)hydrazono)-5fluoroindolin-2-one (6b) - orange powder (yield 73\%), m.p. $>300^{\circ} \mathrm{C}$; IR $\left(\mathrm{KBr}, v \mathrm{~cm}^{-1}\right): 3,420(\mathrm{NH})$ and $1,700(\mathrm{C}=\mathrm{O})$; ${ }^{1} \mathrm{H} \mathrm{NMR}\left(\mathrm{DMSO}-d_{6}\right) \delta \mathrm{ppm}: 4.01\left(\mathrm{~s}, 3 \mathrm{H}, \mathrm{OCH}_{3}\right), 4.03$ (s, 3H, $\left.\mathrm{OCH}_{3}\right), 7.00-7.39$ (m, 3H, Ar-H), 7.73 (s, 1H, Ar-H), 8.07 (s, 1H, Ar-H), 8.72 (s, 1H, Ar-H), 11.33 (s, 1H, NH), 13.65 $(\mathrm{s}, 1 \mathrm{H}, \mathrm{NH}) ;{ }^{13} \mathrm{C} \mathrm{NMR}\left(\mathrm{DMSO}-d_{6}\right) \delta \mathrm{ppm}: 56.0,56.5,104.4$, $108.5,110.4\left({ }^{3} J_{\text {F-C }}=9.0 \mathrm{~Hz}\right), 111.9,\left({ }^{2} J_{\text {F-C }}=27.0 \mathrm{~Hz}\right), 113.0$ $\left({ }^{2} J_{\text {F-C }}=25.5 \mathrm{~Hz}\right), 118.4\left({ }^{2} J_{\text {F-C }}=8.0 \mathrm{~Hz}\right), 120.4,122.3,128.0$, $131.5,133.4,144.4,149.5,153.8\left({ }^{1} J_{\text {F-C }}=238.0 \mathrm{~Hz}\right), 155.5$, 166.2; MS (ESI) m/z: $368.0[\mathrm{M}+\mathrm{H}]^{+}$; Anal. calcd. for $\mathrm{C}_{18} \mathrm{H}_{14} \mathrm{FN}_{5} \mathrm{O}_{3}(367.11)$ : C, 58.85; H, 3.84; N, 19.07; found $\mathrm{C}$, 59.09; H, 3.77; N, 19.13; HRMS (MALDI) calcd. for $\mathrm{C}_{18} \mathrm{H}_{14} \mathrm{FN}_{5} \mathrm{O}_{3}: 368.1159$, found: $368.1151[\mathrm{M}+\mathrm{H}]^{+}$.

5-Chloro-3-(2-(6,7-dimethoxyquinazolin-4-yl)hydrazono)indolin-2-one (6c) - orange powder (yield 80\%), m.p. $>300^{\circ} \mathrm{C}$; IR $\left(\mathrm{KBr}, v \mathrm{~cm}^{-1}\right): 3,421(\mathrm{NH})$ and 1,706 $(\mathrm{C}=\mathrm{O}) ;{ }^{1} \mathrm{H}$ NMR (DMSO- $\left.d_{6}\right) \delta$ ppm: 3.96 (s, 3H, $\mathrm{OCH}_{3}$ ), $4.00\left(\mathrm{~s}, 3 \mathrm{H}, \mathrm{OCH}_{3}\right), 6.90$ (d, 1H, Ar-H, J=8.3 Hz), 7.21 (s, 1H, Ar-H), 7.36 (d, 1H, Ar-H, J=8.3 Hz), 7.72 (s, 1H, Ar-H), 8.08 (s, 1H, Ar-H), 8.57 (s, 1H, Ar-H), 10.71 (s, 1H, $\mathrm{NH}), 12.45$ (s, 1H, NH); ${ }^{13} \mathrm{C}$ NMR (DMSO- $\left.d_{6}\right) \delta$ ppm: 55.7 $\left(\mathrm{OCH}_{3}\right), 56.6\left(\mathrm{OCH}_{3}\right), 104.0,108.5,111.78,112.9,119.7$, 125.7, 127.4, 130.5, 141.9, 142.5, 144.0, 149.5, 152.4, 153.7, 155.4, $166.0(\mathrm{C}=\mathrm{O})$; MS (ESI) $m / z: 384.0[\mathrm{M}+\mathrm{H}]^{+}$; Anal. calcd. for $\mathrm{C}_{18} \mathrm{H}_{14} \mathrm{ClN}_{5} \mathrm{O}_{3}$ (383.08): C, 56.33; H, 3.68; N, 18.25; found C, 56.21; H, 3.72; N, 18.17; HRMS (MALDI) calcd. for $\mathrm{C}_{18} \mathrm{H}_{14} \mathrm{ClN}_{5} \mathrm{O}_{3}: 384.0863$, found: $384.0853[\mathrm{M}+\mathrm{H}]^{+}$.

5-Bromo-3-(2-(6,7-dimethoxyquinazolin-4-yl)hydrazono)indolin-2-one (6d) - orange powder (yield 85\%), m.p. $>300^{\circ} \mathrm{C}$; IR $\left(\mathrm{KBr}, v \mathrm{~cm}^{-1}\right): 3,420(\mathrm{NH})$ and 1,717 $(\mathrm{C}=\mathrm{O}) ;{ }^{1} \mathrm{H} \mathrm{NMR}\left(\mathrm{DMSO}-d_{6}\right) \delta \mathrm{ppm}: 3.96\left(\mathrm{~s}, 3 \mathrm{H}, \mathrm{OCH}_{3}\right), 4.01$ $\left(\mathrm{s}, 3 \mathrm{H}, \mathrm{OCH}_{3}\right), 6.86(\mathrm{~d}, 1 \mathrm{H}, \mathrm{Ar}-\mathrm{H}, J=8.3 \mathrm{~Hz}), 7.21(\mathrm{~s}, 1 \mathrm{H}$, Ar-H), 7.48 (d, 1H, Ar-H, J=8.3 Hz), 7.72 (s, 1H, Ar-H), 8.08 (s, 1H, Ar-H), 8.73 (s, 1H, Ar-H), 10.73 (s, 1H, NH), 12.45 $(\mathrm{s}, 1 \mathrm{H}, \mathrm{NH}) ;{ }^{13} \mathrm{C} \mathrm{NMR}\left(\mathrm{DMSO}-d_{6}\right) \delta \mathrm{ppm}: 55.9\left(\mathrm{OCH}_{3}\right)$, 56.6 $\left(\mathrm{OCH}_{3}\right), 104.0,108.4,112.3,112.9,113.5,120.2,130.3$, 133.3, 142.3, 142.9, 143.6, 144.3, 149.6, 155.5, 155.8, 165.9 $(\mathrm{C}=\mathrm{O})$; MS (ESI) $\mathrm{m} / z$ : $428.0[\mathrm{M}+\mathrm{H}]^{+}$; Anal. calcd. for $\mathrm{C}_{18} \mathrm{H}_{14} \mathrm{BrN}_{5} \mathrm{O}_{3}$ (427.03): C, 50.48; H, 3.30; N, 16.35; found $\mathrm{C}$, 50.61; H, 3.26; N, 16.28; HRMS (MALDI) calcd. for $\mathrm{C}_{18} \mathrm{H}_{14} \mathrm{BrN}_{5} \mathrm{O}_{3}: 428.0358$, found: $428.0353[\mathrm{M}+\mathrm{H}]^{+}$.

3-(2-(6,7-Dimethoxyquinazolin-4-yl)hydrazono)-5methoxyindolin-2-one (6e) - red powder (yield 72\%), m.p. $>300^{\circ} \mathrm{C}$; IR $\left(\mathrm{KBr}, v \mathrm{~cm}^{-1}\right): 3,413(\mathrm{NH})$ and 1,699 $(\mathrm{C}=\mathrm{O})$; ${ }^{1} \mathrm{H} \mathrm{NMR}\left(\mathrm{DMSO}-d_{6}\right) \delta \mathrm{ppm}: 3.81\left(\mathrm{~s}, 3 \mathrm{H}, \mathrm{OCH}_{3}\right), 3.93(\mathrm{~s}, 3 \mathrm{H}$, $\left.\mathrm{OCH}_{3}\right), 3.95\left(\mathrm{~s}, 3 \mathrm{H}, \mathrm{OCH}_{3}\right), 6.80(\mathrm{~d}, 1 \mathrm{H}, \mathrm{Ar}-\mathrm{H}, J=8.4 \mathrm{~Hz})$, 6.90 (d, 1H, Ar-H, J=8.4 Hz), 7.19 (s, 1H, Ar-H), 7.76 (s, 1H, Ar-H), 8.06 (s, 1H, Ar-H), 8.71 (s, 1H, Ar-H), 10.40 $(\mathrm{s}, 1 \mathrm{H}, \mathrm{NH}), 12.23(\mathrm{~s}, 1 \mathrm{H}, \mathrm{NH}) ;{ }^{13} \mathrm{C} \mathrm{NMR}\left(\mathrm{DMSO}-d_{6}\right) \delta \mathrm{ppm}$ : $55.6\left(\mathrm{OCH}_{3}\right), 55.7\left(\mathrm{OCH}_{3}\right), 56.5\left(\mathrm{OCH}_{3}\right), 104.2,108.6,111.1$, 112.5, 113.0, 118.0, 118.7, 137.2, 143.2, 143.7, 144.1, 149.4, 154.1, 154.9, 155.2, $166.4(\mathrm{C}=\mathrm{O})$; MS (ESI) $m / z: 380.0$ $[\mathrm{M}+\mathrm{H}]^{+}$; Anal. calcd. for $\mathrm{C}_{19} \mathrm{H}_{17} \mathrm{~N}_{5} \mathrm{O}_{4}$ (379.13): C, 60.15; $\mathrm{H}, 4.52$; N, 18.46; found $\mathrm{C}, 59.91 ; \mathrm{H}, 4.58 ; \mathrm{N}, 18.59$; HRMS (MALDI) calcd. for $\mathrm{C}_{19} \mathrm{H}_{17} \mathrm{~N}_{5} \mathrm{O}_{4}: 380.1359$, found: $380.1345[\mathrm{M}+\mathrm{H}]^{+}$.

3-(2-(6,7-Dimethoxyquinazolin-4-yl)hydrazono)-5methylindolin-2-one (6f) - orange powder (yield 75\%), m.p. $>300^{\circ} \mathrm{C}$; IR $\left(\mathrm{KBr}, v \mathrm{~cm}^{-1}\right): 3,421(\mathrm{NH})$ and 1,700 $(\mathrm{C}=\mathrm{O})$; ${ }^{1} \mathrm{H}$ NMR (DMSO- $\left.d_{6}\right) \delta$ ppm: $2.31\left(\mathrm{~s}, 3 \mathrm{H}, \mathrm{CH}_{3}\right.$ ), 3.95 (s, 3H, $\left.\mathrm{OCH}_{3}\right), 4.01$ (s, 3H, OCH $), 6.77$ (d, 1H, Ar-H, J=7.5 Hz), 7.12 (d, 1H, Ar-H, J=7.0 Hz), 7.18 (s, 1H, Ar-H), 7.77 (s, 1H, Ar-H), 8.02 (s, 1H, Ar-H), 8.69 (s, 1H, Ar-H), 10.48 $(\mathrm{s}, 1 \mathrm{H}, \mathrm{NH}), 12.32(\mathrm{~s}, 1 \mathrm{H}, \mathrm{NH}) ;{ }^{13} \mathrm{C} \mathrm{NMR}\left(\mathrm{DMSO}-d_{6}\right) \delta \mathrm{ppm}$ : $21.3\left(\mathrm{CH}_{3}\right), 55.7\left(\mathrm{OCH}_{3}\right), 56.5\left(\mathrm{OCH}_{3}\right), 104.2,108.6,110.1$, $113.1,118.6,128.9,130.8,131.8,141.2,143.8,144.8$, 149.4, 153.3, 155.1, 156.3, $166.4(\mathrm{C}=\mathrm{O})$; MS (ESI) $\mathrm{m} / \mathrm{z}$ : $364.0[\mathrm{M}+\mathrm{H}]^{+}$; Anal. calcd. for $\mathrm{C}_{19} \mathrm{H}_{17} \mathrm{~N}_{5} \mathrm{O}_{3}$ (363.13): C, 62.80; H, 4.72; N, 19.27; found C, 63.03; H, 4.66; N, 19.15; HRMS (MALDI) calcd. for $\mathrm{C}_{19} \mathrm{H}_{17} \mathrm{~N}_{5} \mathrm{O}_{3}: 364.1409$, found: $364.1422[\mathrm{M}+\mathrm{H}]^{+}$.

$3-(2-(2-(3,4-D i m e t h o x y p h e n y l) q u i n a z o l i n-4-y 1)$ hydrazono)indolin-2-one (7a). ${ }^{35}$

$3-(2-(2-(3,4-D i m e t h o x y p h e n y l) q u i n a z o l i n-4-y 1)$ hydrazono)-5-fluoroindolin-2-one (7b) ${ }^{35}$

5-Chloro-3-(2-(2-(3,4-dimethoxyphenyl)quinazolin-4-yl) hydrazono)indolin-2-one (7c) ${ }^{35}$

5-Bromo-3-(2-(2-(3,4-dimethoxyphenyl)quinazolin-4-yl) hydrazono)indolin-2-one (7d) - red powder (yield 80\%), m.p. $295^{\circ} \mathrm{C}-297^{\circ} \mathrm{C}$; IR $\left(\mathrm{KBr}, v \mathrm{~cm}^{-1}\right)$ : 3,421 (NH) and 1,718 $(\mathrm{C}=\mathrm{O}) ;{ }^{1} \mathrm{H}$ NMR (DMSO-d $\left.{ }_{6}\right) \delta$ ppm: $3.87\left(\mathrm{~s}, 3 \mathrm{H}, \mathrm{OCH}_{3}\right)$, $3.92\left(\mathrm{~s}, 3 \mathrm{H}, \mathrm{OCH}_{3}\right), 6.96(\mathrm{~d}, 1 \mathrm{H}, \mathrm{Ar}-\mathrm{H}, J=8.0 \mathrm{~Hz}), 7.15$ (d, 1H, Ar-H, $J=8.0 \mathrm{~Hz}$ ), 7.55 (d, 1H, Ar-H, $J=8.5 \mathrm{~Hz}$ ), 7.73 (t, 1H, Ar-H, J=7.0 Hz), 7.85 (s, 1H, Ar-H), 7.94-7.97 $(\mathrm{m}, 2 \mathrm{H}, \mathrm{Ar}-\mathrm{H}), 8.12$ (s, 1H, Ar-H), 8.18 (d, 1H, Ar-H, $J=8.5 \mathrm{~Hz}), 8.52-8.53(\mathrm{~m}, 1 \mathrm{H}, \mathrm{Ar}-\mathrm{H}), 11.49$ (s, 1H, NH), $13.81(\mathrm{~s}, 1 \mathrm{H}, \mathrm{NH}) ;{ }^{13} \mathrm{C} \mathrm{NMR}\left(\mathrm{DMSO}-d_{6}\right) \delta \mathrm{ppm}: 56.2$ $\left(\mathrm{OCH}_{3}\right), 56.3\left(\mathrm{OCH}_{3}\right), 110.7,110.9,112.1,118.1,120.6$, 122.4, 125.1, 125.3, 127.7, 128.0, 128.4, 132.3, 132.5, 134.9, 143.3, 146.0, 147.4, 149.3, 152.5, $166.2(\mathrm{C}=\mathrm{O})$; MS (ESI) $m / z: 504[\mathrm{M}+\mathrm{H}]^{+}$; Anal. calcd. for $\mathrm{C}_{24} \mathrm{H}_{18} \mathrm{BrN}_{5} \mathrm{O}_{3}$ (503.06): 
C, 57.16; H, 3.60; N, 13.89; found C, 57.29; H, 3.63; N, 13.79; HRMS (MALDI) calcd. for $\mathrm{C}_{24} \mathrm{H}_{18} \mathrm{BrN}_{5} \mathrm{O}_{3}: 504.0671$, found: $504.0653[\mathrm{M}+\mathrm{H}]^{+}$.

3-(2-(2-(3,4-Dimethoxyphenyl)quinazolin-4-yl) hydrazono)-5-methoxyindolin-2-one (7e) - orange powder (yield 79\%), m.p. $263^{\circ} \mathrm{C}-265^{\circ} \mathrm{C}$; IR $\left(\mathrm{KBr}, v \mathrm{~cm}^{-1}\right): 3,412$ $(\mathrm{NH})$ and $1,718(\mathrm{C}=\mathrm{O}) ;{ }^{1} \mathrm{H}$ NMR (DMSO- $\left.d_{6}\right) \delta \mathrm{ppm}$ : $3.76\left(\mathrm{~s}, 3 \mathrm{H}, \mathrm{OCH}_{3}\right), 3.89\left(\mathrm{~s}, 3 \mathrm{H}, \mathrm{OCH}_{3}\right), 3.91$ (s, 3H, OCH $)_{3}$, $6.83(\mathrm{~d}, 1 \mathrm{H}, \mathrm{Ar}-\mathrm{H}, J=8.5 \mathrm{~Hz}), 6.96$ (d, 1H, Ar-H, $J=8.5 \mathrm{~Hz})$, 7.14 (s, 1H, Ar-H), 7.19 (d, 1H, Ar-H, $J=9.0$ Hz), 7.81 (t, 1H, Ar-H, $J=7.5 \mathrm{~Hz}$ ), 7.96 (s, 1H, Ar-H), 8.06-8.09 (m, 4H, Ar-H), 11.31 (s, 1H, NH), 13.08 (s, 1H, NH); ${ }^{13} \mathrm{C}$ NMR (DMSO- $\left.d_{6}\right) \delta$ ppm: $55.8\left(\mathrm{OCH}_{3}\right), 56.2\left(\mathrm{OCH}_{3}\right), 56.3$ $\left(\mathrm{OCH}_{3}\right), 110.0,110.8,111.5,113.3,114.4,117.6,120.9$, 123.1, 124.8, 125.8, 127.1, 127.9, 129.1, 131.8, 133.0, 135.2, $142.5,144.7,148.2,149.1,154.8,166.3(\mathrm{C}=\mathrm{O})$; MS (ESI) $m / z: 456[\mathrm{M}+\mathrm{H}]^{+}$; Anal. calcd. for $\mathrm{C}_{25} \mathrm{H}_{21} \mathrm{~N}_{5} \mathrm{O}_{4}$ (455.16): C, 65.93; H, 4.65; N, 15.38; found C, 66.17; H, 4.59; $\mathrm{N}, 15.52$; HRMS (MALDI) calcd. for $\mathrm{C}_{25} \mathrm{H}_{21} \mathrm{~N}_{5} \mathrm{O}_{4}: 456.1672$, found: $456.1662[\mathrm{M}+\mathrm{H}]^{+}$.

3-(2-(4-Benzylphthalazin-1-yl)hydrazono)indolin-2one (8a) - orange powder (yield $75 \%$ ), m.p. $259^{\circ} \mathrm{C}-261^{\circ} \mathrm{C}$; IR (KBr, $\left.v \mathrm{~cm}^{-1}\right): 3,412(\mathrm{NH})$ and 1,700 $(\mathrm{C}=\mathrm{O}) ;{ }^{1} \mathrm{H}$ NMR (DMSO- $\left.d_{6}\right) \delta$ ppm: $4.36\left(\mathrm{~s}, 2 \mathrm{H}, \mathrm{CH}_{2}\right), 6.89$ (d, 1H, Ar-H, $J=7.5 \mathrm{~Hz}), 7.06(\mathrm{t}, 1 \mathrm{H}, \mathrm{Ar}-\mathrm{H}, J=7.5 \mathrm{~Hz}), 7.19(\mathrm{t}, 1 \mathrm{H}$, Ar-H, $J=7.5 \mathrm{~Hz}), 7.29-7.36(\mathrm{~m}, 5 \mathrm{H}, \mathrm{Ar}-\mathrm{H}), 7.86-7.98$ (m, 3H, Ar-H), 8.45 (d, 1H, Ar-H, J=7.5 Hz), 8.63 (d, 1H, Ar-H, $J=7.5 \mathrm{~Hz}$ ), 10.63 (s, 1H, NH), 12.89 (s, 1H, NH); ${ }^{13} \mathrm{C}$ NMR (DMSO- $\left.d_{6}\right) \delta$ ppm: $38.1\left(\mathrm{CH}_{2}\right), 111.6,115.6$, $118.3,120.3,122.3,126.2,127.0,127.6,127.8,128.9,129.1$, 131.4, 132.9, 134.0, 138.6, 143.3, 144.0, 148.4, 156.5, 166.7; MS (ESI) $m / z: 380.0[\mathrm{M}+\mathrm{H}]^{+}$; Anal. calcd. for $\mathrm{C}_{23} \mathrm{H}_{17} \mathrm{~N}_{5} \mathrm{O}$ (379.14): C, 72.81; H, 4.52; N, 18.46; found C, 73.01; $\mathrm{H}, 4.48$; N, 18.55; HRMS (MALDI) calcd. for $\mathrm{C}_{23} \mathrm{H}_{17} \mathrm{~N}_{5} \mathrm{O}$ : 380.1511, found: $380.1525[\mathrm{M}+\mathrm{H}]^{+}$.

3-(2-(4-Benzylphthalazin-1-yl)hydrazono)-5-fluoroindolin-2-one $(\mathbf{8 b})$ - orange powder (yield 79\%), m.p. $275^{\circ} \mathrm{C}-277^{\circ} \mathrm{C}$; IR $\left(\mathrm{KBr}, v \mathrm{~cm}^{-1}\right): 3,411(\mathrm{NH})$ and 1,705 $(\mathrm{C}=\mathrm{O}) ;{ }^{1} \mathrm{H}$ NMR (DMSO- $\left.d_{6}\right) \delta$ ppm: 4.39 (s, 2H, $\mathrm{CH}_{2}$ ), 6.88-7.36 (m, 7H, Ar-H), 7.90-8.01 (m, 3H, Ar-H), 8.19 $(\mathrm{d}, 1 \mathrm{H}, \mathrm{Ar}-\mathrm{H}, J=8.5 \mathrm{~Hz}), 8.61$ (d, $1 \mathrm{H}, \mathrm{Ar}-\mathrm{H}, J=7.0 \mathrm{~Hz}), 10.62$ (s, $1 \mathrm{H}, \mathrm{NH}), 12.93(\mathrm{~s}, 1 \mathrm{H}, \mathrm{NH}) ;{ }^{13} \mathrm{C}$ NMR (DMSO- $\left.d_{6}\right) \delta \mathrm{ppm}$ : $38.1\left(\mathrm{CH}_{2}\right), 111.1\left({ }^{3} J_{\mathrm{F}-\mathrm{C}}=8.8 \mathrm{~Hz}\right), 114.0\left({ }^{2} J_{\mathrm{F}-\mathrm{C}}=24.5 \mathrm{~Hz}\right), 117.3$ $\left({ }^{2} J_{\text {F-C }}=25.0 \mathrm{~Hz}\right), 118.7\left({ }^{3} J_{\text {F-C }}=8.8 \mathrm{~Hz}\right), 125.9,126.2,126.6$, 127.0, 127.6, 128.9, 129.1, 133.1, 134.1, 138.6, 139.4, 143.3, 148.9, 152.8, $157.3\left({ }^{1} J_{\text {F-C }}=234.5 \mathrm{~Hz}\right), 166.5$; MS (ESI) $m / z: 398.0[\mathrm{M}+\mathrm{H}]^{+}$; Anal. calcd. for $\mathrm{C}_{23} \mathrm{H}_{16} \mathrm{FN}_{5} \mathrm{O}$ (397.13): C, 69.51; H, 4.06; N, 17.62; found $\mathrm{C}, 69.32 ; \mathrm{H}, 4.13$;
$\mathrm{N}, 17.51$; HRMS (MALDI) calcd. for $\mathrm{C}_{23} \mathrm{H}_{16} \mathrm{FN}_{5} \mathrm{O}: 398.1417$, found: $398.1405[\mathrm{M}+\mathrm{H}]^{+}$.

3-(2-(4-Benzylphthalazin-1-yl)hydrazono)-5-chloroindolin-2-one $(\mathbf{8 c})$ - orange powder (yield 80\%), m.p. $295^{\circ} \mathrm{C}-297^{\circ} \mathrm{C}$; IR $\left(\mathrm{KBr}, v \mathrm{~cm}^{-1}\right): 3,410(\mathrm{NH})$ and 1,700 $(\mathrm{C}=\mathrm{O}) ;{ }^{1} \mathrm{H}$ NMR (DMSO- $\left.d_{6}\right) \delta$ ppm: 4.39 (s, 2H, $\mathrm{CH}_{2}$ ), 6.91 (d, 1H, Ar-H, $J=8.5 \mathrm{~Hz}), 7.21$ (t, $1 \mathrm{H}, \operatorname{Ar}-\mathrm{H}, J=7.5 \mathrm{~Hz}$ ), 7.30-7.36 (m, 5H, Ar-H), 7.90-8.02 (m, 3H, Ar-H), 8.42 (s, 1H, Ar-H), 8.56 (d, 1H, Ar-H, J=7.0 Hz), 10.73 (s, 1H, $\mathrm{NH}), 12.96(\mathrm{~s}, 1 \mathrm{H}, \mathrm{NH}) ;{ }^{13} \mathrm{C}$ NMR (DMSO- $\left.d_{6}\right) \delta \mathrm{ppm}: 38.1$ $\left(\mathrm{CH}_{2}\right), 111.8,119.5,125.7,126.3,126.6,126.7,127.0$, $127.7,128.9,129.0,129.1,130.5,133.1,134.2,138.6$, 141.8, 142.7, 149.0, 152.8, $166.2(\mathrm{C}=\mathrm{O})$; MS (ESI) $m / z$ : $414.0[\mathrm{M}+\mathrm{H}]^{+}$; Anal. calcd. for $\mathrm{C}_{23} \mathrm{H}_{16} \mathrm{ClN}_{5} \mathrm{O}$ (413.10): C, 66.75; H, 3.90; N, 16.92; found C, 66.97; H, 3.83; N, 17.05; HRMS (MALDI) calcd. for $\mathrm{C}_{23} \mathrm{H}_{16} \mathrm{ClN}_{5} \mathrm{O}: 414.1122$, found: $414.1146[\mathrm{M}+\mathrm{H}]^{+}$.

3-(2-(4-Benzylphthalazin-1-yl)hydrazono)-5-bromoindolin-2-one (8d) - orange powder (yield 86\%), m.p. $298^{\circ} \mathrm{C}-299^{\circ} \mathrm{C}$; IR $\left(\mathrm{KBr}, v \mathrm{~cm}^{-1}\right): 3,412(\mathrm{NH})$ and 1,716 $(\mathrm{C}=\mathrm{O}) ;{ }^{1} \mathrm{H}$ NMR (DMSO-d $)$ ) $\delta$ ppm: $4.39\left(\mathrm{~s}, 2 \mathrm{H}, \mathrm{CH}_{2}\right), 6.86$ (d, 1H, Ar-H, $J=8.5 \mathrm{~Hz}), 7.21(\mathrm{t}, 1 \mathrm{H}, \operatorname{Ar}-\mathrm{H}, J=7.5 \mathrm{~Hz})$, 7.30-7.36 (m, 3H, Ar-H), 7.47 (d, 1H, Ar-H, $J=8.5$ Hz), 7.90-8.02 (m, 3H, Ar-H), 8.20 (s, 1H, Ar-H), 8.53-8.56 (m, 2H, Ar-H), 10.74 (s, 1H, NH), 12.69 (s, 1H, NH); ${ }^{13} \mathrm{C}$ NMR (DMSO- $\left.d_{6}\right) \delta$ ppm: $38.1\left(\mathrm{CH}_{2}\right), 112.3,113.5,120.0$, 126.4, 126.6, 127.0, 127.7, 128.8, 128.9, 129.1, 129.5, 133.0, $133.3,134.2$, 138.5 , 142.1 142.6, 149.0, 152.8, $166.0(\mathrm{C}=\mathrm{O})$; MS (ESI) $m / z: 458.0[\mathrm{M}+\mathrm{H}]^{+}$; Anal. calcd. for $\mathrm{C}_{23} \mathrm{H}_{16} \mathrm{BrN}_{5} \mathrm{O}$ (457.05): C, 60.28; H, 3.52; N, 15.28; found C, 60.46; $\mathrm{H}, 3.47$; N, 15.40; HRMS (MALDI) calcd. for $\mathrm{C}_{23} \mathrm{H}_{16} \mathrm{BrN}_{5} \mathrm{O}$ : 458.0617, found: $458.0605[\mathrm{M}+\mathrm{H}]^{+}$.

3-(2-(4-Benzylphthalazin-1-yl)hydrazono)-5-methoxyindolin-2-one $(\mathbf{8 e})$ - red powder (yield $74 \%$ ), m.p. $>300^{\circ} \mathrm{C}$; IR $\left(\mathrm{KBr}, v \mathrm{~cm}^{-1}\right): 3,420(\mathrm{NH})$ and $1,707(\mathrm{C}=\mathrm{O}) ;{ }^{1} \mathrm{H}$ NMR (DMSO- $\left.d_{6}\right) \delta$ ppm: $3.79\left(\mathrm{~s}, 3 \mathrm{H}, \mathrm{OCH}_{3}\right), 4.37$ $\left(\mathrm{s}, 2 \mathrm{H}, \mathrm{CH}_{2}\right), 6.80$ (d, 1H, Ar-H, $\left.J=8.5 \mathrm{~Hz}\right), 6.91(\mathrm{~d}, 1 \mathrm{H}$, Ar-H, $J=8.5 \mathrm{~Hz}$ ), 7.19 (t, 1H, Ar-H, $J=7.5 \mathrm{~Hz}), 7.29-7.36$ (m, 4H, Ar-H), 7.87-7.99 (m, 3H, Ar-H), 8.08 (s, 1H, Ar-H), 8.56 (d, 1H, Ar-H, J=8.0 Hz), 10.44 (s, 1H, NH), 12.88 $(\mathrm{s}, 1 \mathrm{H}, \mathrm{NH}) ;{ }^{13} \mathrm{C}$ NMR (DMSO- $\left.d_{6}\right) \delta \mathrm{ppm}: 38.1\left(\mathrm{CH}_{2}\right), 55.8$ $\left(\mathrm{OCH}_{3}\right), 110.7,112.1,113.5,116.9,120.1,123.7,126.4$, $127.1,127.6,128.8,129.0,129.2,131.7,133.8,138.5$, 141.7, 144.2, 146.2, 149.9, 154.5, $166.5(\mathrm{C}=\mathrm{O})$; MS (ESI) $m / z: 410.0[\mathrm{M}+\mathrm{H}]^{+}$; Anal. calcd. for $\mathrm{C}_{24} \mathrm{H}_{19} \mathrm{~N}_{5} \mathrm{O}_{2}$ (409.15): C, 70.40; H, 4.68; N, 17.10; found C, 70.64; H, 4.63; N, 16.98; HRMS (MALDI) calcd. for $\mathrm{C}_{24} \mathrm{H}_{19} \mathrm{~N}_{5} \mathrm{O}_{2}: 410.1617$, found: $410.1644[\mathrm{M}+\mathrm{H}]^{+}$. 
3-(2-(4-Benzylphthalazin-1-yl)hydrazono)-5-methylindolin-2-one (8f) - orange powder (yield 71\%), m.p. $253^{\circ} \mathrm{C}-255^{\circ} \mathrm{C}$; IR (KBr, $\left.v \mathrm{~cm}^{-1}\right): 3,350(\mathrm{NH})$ and 1,697 $(\mathrm{C}=\mathrm{O}) ;{ }^{1} \mathrm{H}$ NMR $\left(\mathrm{DMSO}-d_{6}\right) \delta$ ppm: $2.34\left(\mathrm{~s}, 3 \mathrm{H}, \mathrm{CH}_{3}\right), 4.36$ $\left(\mathrm{s}, 2 \mathrm{H}, \mathrm{CH}_{2}\right), 6.78$ (d, 1H, Ar-H, J=7.5 Hz), 7.12 (d, 1H, Ar-H, $J=8.0 \mathrm{~Hz}$ ), 7.21 (t, 1H, Ar-H, $J=7.5 \mathrm{~Hz}), 7.29-7.36$ (m, 4H, Ar-H), 7.88-7.97 (m, 3H, Ar-H), 8.26 (s, 1H, Ar-H), 8.60 (d, 1H, Ar-H, J=7.5 Hz), 10.52 (s, 1H, NH), 12.84 $(\mathrm{s}, 1 \mathrm{H}, \mathrm{NH}) ;{ }^{13} \mathrm{C} \mathrm{NMR}\left(\mathrm{DMSO}-d_{6}\right) \delta \mathrm{ppm}: 21.4\left(\mathrm{CH}_{3}\right), 38.1$ $\left(\mathrm{CH}_{2}\right), 110.1,118.5,125.8,126.1,126.8,127.0,127.5,128.3$, 128.9, 129.1, 130.8, 131.7, 132.8, 133.8, 138.6, 141.2, 144.6, 148.2, 152.1, 166.6(C=O); MS (ESI) $m / z: 394.0[\mathrm{M}+\mathrm{H}]^{+}$; Anal. calcd. for $\mathrm{C}_{24} \mathrm{H}_{19} \mathrm{~N}_{5} \mathrm{O}$ (393.16): C, 73.27; H, 4.87; N, 17.80; found C, 73.39; H, 4.91; N, 17.72; HRMS (MALDI) calcd. for $\mathrm{C}_{24} \mathrm{H}_{19} \mathrm{~N}_{5} \mathrm{O}$ : 394.1668, found: $394.1646[\mathrm{M}+\mathrm{H}]^{+}$.

1-Methyl-3-(2-(2-oxoindolin-3-ylidene)hydrazinyl) quinoxalin-2(1H)-one (9a) - orange powder (yield 80\%), m.p. $>300^{\circ} \mathrm{C}$; IR $\left(\mathrm{KBr}, v \mathrm{~cm}^{-1}\right): 3,347(\mathrm{NH})$ and 1,711 $(\mathrm{C}=\mathrm{O}) ;{ }^{1} \mathrm{H}$ NMR $\left(\mathrm{DMSO}-d_{6}\right) \delta$ ppm: $3.71\left(\mathrm{~s}, 3 \mathrm{H}, \mathrm{CH}_{3}\right), 6.95$ (d, 1H, Ar-H, $J=7.5 \mathrm{~Hz}), 7.10$ (t, 1H, Ar-H, $J=7.5 \mathrm{~Hz}$ ), 7.33-7.39 (m, 2H, Ar-H), 7.46 (t, 1H, Ar-H, J=7.5 Hz), 7.54 (d, 1H, Ar-H, $J=8.0 \mathrm{~Hz}$ ), 7.64 (d, 1H, Ar-H, $J=7.5 \mathrm{~Hz}$ ), 7.71 (d, 1H, Ar-H, $J=7.5 \mathrm{~Hz}$ ), 10.72 (s, 1H, NH), 11.24 $(\mathrm{s}, 1 \mathrm{H}, \mathrm{NH}) ;{ }^{13} \mathrm{C}$ NMR (DMSO- $\left.d_{6}\right) \delta \mathrm{ppm}: 29.8\left(\mathrm{CH}_{3}\right)$, 111.5, 115.3, 120.1, 121.0, 122.9, 124.7, 126.6, 131.5, 132.6, $137.8,140.5,142.0,143.2,150.5,162.3,168.3$; MS (ESI) $m / z: 320.0[\mathrm{M}+\mathrm{H}]^{+}$; Anal. calcd. for $\mathrm{C}_{17} \mathrm{H}_{13} \mathrm{~N}_{5} \mathrm{O}_{2}$ (319.11): C, 63.94; H, 4.10; N, 21.93; found C, 64.17; H, 4.07; N, 21.84; HRMS (MALDI) calcd. for $\mathrm{C}_{17} \mathrm{H}_{13} \mathrm{~N}_{5} \mathrm{O}_{2}: 320.1148$, found: $320.1133[\mathrm{M}+\mathrm{H}]^{+}$.

3-(2-(5-Fluoro-2-oxoindolin-3-ylidene)hydrazinyl)-1methylquinoxalin-2(1H)-one (9b) - red powder (yield 75\%), m.p. $>300^{\circ} \mathrm{C}$; IR $\left(\mathrm{KBr}, v \mathrm{~cm}^{-1}\right): 3,412(\mathrm{NH})$ and 1,701 $(\mathrm{C}=\mathrm{O}) ;{ }^{1} \mathrm{H}$ NMR (DMSO- $\left.d_{6}\right) \delta$ ppm: $3.71\left(\mathrm{~s}, 3 \mathrm{H}, \mathrm{CH}_{3}\right)$, 6.94-6.97 (m, 1H, Ar-H), 7.18 (t, 1H, Ar-H, J=7.0 Hz), 7.37 (t, 1H, Ar-H, J=7.5 Hz), 7.43-7.50 (m, 2H, Ar-H), 7.55 (d, 1H, Ar-H, J=8.0 Hz), 7.71 (d, 1H, Ar-H, J=7.5 Hz), 11.26 (s, 1H, NH), $13.70(\mathrm{~s}, 1 \mathrm{H}, \mathrm{NH}) ;{ }^{13} \mathrm{C} \mathrm{NMR}\left(\mathrm{DMSO}-d_{6}\right) \delta \mathrm{ppm}$ : $29.7\left(\mathrm{CH}_{3}\right), 111.4\left({ }^{3} J_{\mathrm{F}-\mathrm{C}}=9.0 \mathrm{~Hz}\right), 115.2\left({ }^{2} J_{\mathrm{F}-\mathrm{C}}=28.8 \mathrm{~Hz}\right), 118.5$ $\left({ }^{2} J_{\mathrm{F}-\mathrm{C}}=28.00 \mathrm{~Hz}\right), 119.0\left({ }^{3} J_{\mathrm{F}-\mathrm{C}}=9.0 \mathrm{~Hz}\right), 121.9,123.9,125.7$, $128.2,131.5,138.3,140.4,147.1,150.8,156.8\left({ }^{1} J_{\text {F-C }}=239.5\right.$ $\mathrm{Hz}), 163.5,165.9$; MS (ESI) m/z: $338.0[\mathrm{M}+\mathrm{H}]^{+}$; Anal. calcd. for $\mathrm{C}_{17} \mathrm{H}_{12} \mathrm{FN}_{5} \mathrm{O}_{2}$ (337.10): C, 60.53; H, 3.59; N, 20.76; found C, 60.74; H, 3.62; N, 20.67; HRMS (MALDI) calcd. for $\mathrm{C}_{17} \mathrm{H}_{12} \mathrm{FN}_{5} \mathrm{O}_{2}: 338.1053$, found: $338.1041[\mathrm{M}+\mathrm{H}]^{+}$.

3-(2-(5-Chloro-2-oxoindolin-3-ylidene)hydrazinyl)-1methylquinoxalin-2(1H)-one $(9 \mathrm{c})$ - orange powder (yield $81 \%$ ), m.p. $>300^{\circ} \mathrm{C}$; IR $\left(\mathrm{KBr}, v^{-1}\right): 3,411(\mathrm{NH})$ and 1,698 $(\mathrm{C}=\mathrm{O}) ;{ }^{1} \mathrm{H}$ NMR $\left(\mathrm{DMSO}-d_{6}\right) \delta$ ppm: $3.71\left(\mathrm{~s}, 3 \mathrm{H}, \mathrm{CH}_{3}\right), 6.97$ (d, 1H, Ar-H, J=8.5 Hz), 7.38-7.41 (m, 2H, Ar-H), $7.50(\mathrm{t}, 1 \mathrm{H}$, Ar-H, $J=7.5 \mathrm{~Hz}$ ), 7.56 (d, 1H, Ar-H, $J=8.5 \mathrm{~Hz}), 7.62$ (s, 1H, Ar-H), 7.72 (d, 1H, Ar-H, J=8.0 Hz), 11.37 (s, 1H, NH), 13.65 (s, $1 \mathrm{H}, \mathrm{NH}),{ }^{13} \mathrm{C} \mathrm{NMR}\left(\mathrm{DMSO}-d_{6}\right) \delta \mathrm{ppm}: 29.8\left(\mathrm{CH}_{3}\right), 112.1$, 115.4, 117.5, 120.3, 122.8, 124.7, 126.1, 128.3, 130.7, 132.1, 134.7, 140.9, 142.7, 150.9, 163.3, 165.5; MS (ESI) $m / z: 354.0$ $[\mathrm{M}+\mathrm{H}]^{+}$; Anal. calcd. for $\mathrm{C}_{17} \mathrm{H}_{12} \mathrm{ClN}_{5} \mathrm{O}_{2}$ (353.07): C, 57.72; $\mathrm{H}, 3.42$; N, 19.80; found C, 57.88; H, 3.38; N, 19.91; HRMS (MALDI) calcd. for $\mathrm{C}_{17} \mathrm{H}_{12} \mathrm{ClN}_{5} \mathrm{O}_{2}: 354.0758$, found: $354.0757[\mathrm{M}+\mathrm{H}]^{+}$.

3-(2-(5-Bromo-2-oxoindolin-3-ylidene)hydrazinyl)-1methylquinoxalin-2(1H)-one (9d) - orange powder (yield $84 \%)$, m.p. $>300^{\circ} \mathrm{C}$; IR $\left(\mathrm{KBr}, v \mathrm{~cm}^{-1}\right): 3,411(\mathrm{NH})$ and 1,708 (C=O); ${ }^{1} \mathrm{H}$ NMR (DMSO- $\left.d_{6}\right) \delta$ ppm: $3.72\left(\mathrm{~s}, 3 \mathrm{H}, \mathrm{CH}_{3}\right.$ ), $6.93(\mathrm{~d}, 1 \mathrm{H}, \mathrm{Ar}-\mathrm{H}, J=8.5 \mathrm{~Hz}), 7.38$ (t, $1 \mathrm{H}, \mathrm{Ar}-\mathrm{H}, J=7.5 \mathrm{~Hz}$ ), 7.48-7.58 (m, 3H, Ar-H), 7.73-7.74 (m, 2H, Ar-H), 11.44 $(\mathrm{s}, 1 \mathrm{H}, \mathrm{NH}), 13.64(\mathrm{~s}, 1 \mathrm{H}, \mathrm{NH}),{ }^{13} \mathrm{C} \mathrm{NMR}\left(\mathrm{DMSO}-d_{6}\right) \delta \mathrm{ppm}$ : $29.8\left(\mathrm{CH}_{3}\right), 112.6,115.4,117.1,119.7,122.8,124.0,127.6$, 128.3, 131.2, 133.4, 141.2, 143.1, 147.8, 150.8, 163.1, 165.4; MS (ESI) $m / z: 398.0[\mathrm{M}+\mathrm{H}]^{+}$; Anal. calcd. for $\mathrm{C}_{17} \mathrm{H}_{12} \mathrm{BrN}_{5} \mathrm{O}_{2}$ (397.02): C, 51.27; H, 3.04; N, 17.59; found C, 51.09; $\mathrm{H}, 3.07$; N, 17.70; HRMS (MALDI) calcd. for $\mathrm{C}_{17} \mathrm{H}_{12} \mathrm{BrN}_{5} \mathrm{O}_{2}$ : 398.0253, found: $398.0243[\mathrm{M}+\mathrm{H}]^{+}$.

3-(2-(5-Methoxy-2-oxoindolin-3-ylidene)hydrazinyl)1-methylquinoxalin-2(1H)-one $(9 e)$ - red powder (yield $78 \%$ ), m.p. $>300^{\circ} \mathrm{C}$; IR $\left(\mathrm{KBr}, v \mathrm{~cm}^{-1}\right): 3,413(\mathrm{NH})$ and 1,707 (C=O); ${ }^{1} \mathrm{H}$ NMR (DMSO- $\left.d_{6}\right) \delta$ ppm: 3.69 (s, 3H, $\mathrm{CH}_{3}$ ), 3.79 (s, 3H, $\left.\mathrm{OCH}_{3}\right), 6.80-6.94(\mathrm{~m}, 2 \mathrm{H}, \mathrm{Ar}-\mathrm{H}), 7.14-7.51$ (m, 4H, Ar-H), 7.69 (d, 1H, Ar-H, J=8.0 Hz), 10.53 (s, 1H, $\mathrm{NH}), 13.70$ (s, 1H, NH); ${ }^{13} \mathrm{C}$ NMR (DMSO- $\left.d_{6}\right) \delta$ ppm: 38.1 $\left(\mathrm{CH}_{3}\right), 55.8\left(\mathrm{OCH}_{3}\right), 110.8,113.6,116.5,118.9,125.5$, 126.8, 127.6, 128.9, 129.1, 132.9, 133.9, 137.0, 138.6, 148.4, 154.9, 166.5; MS (ESI) $m / z: 350.0[\mathrm{M}+\mathrm{H}]^{+}$; Anal. calcd. for $\mathrm{C}_{18} \mathrm{H}_{15} \mathrm{~N}_{5} \mathrm{O}_{3}$ (349.12): C, 61.89; H, 4.33; N, 20.05; found C, 62.11; H, 4.29; N, 19.94; HRMS (MALDI) calcd. for $\mathrm{C}_{18} \mathrm{H}_{15} \mathrm{~N}_{5} \mathrm{O}_{3}: 350.1253$, found: $350.1239[\mathrm{M}+\mathrm{H}]^{+}$.

1-Methyl-3-(2-(5-methyl-2-oxoindolin-3-ylidene) hydrazinyl)quinoxalin-2(1H)-one $(\mathbf{9 f})$ - orange powder (yield 77\%), m.p. $>300^{\circ} \mathrm{C}$; IR $\left(\mathrm{KBr}, \mathrm{v} \mathrm{cm}^{-1}\right)$ : 3,411 (NH) and 1,700 $(\mathrm{C}=\mathrm{O}) ;{ }^{1} \mathrm{H}$ NMR (DMSO- $\left.d_{6}\right) \delta \mathrm{ppm}: 3.70(\mathrm{~s}, 3 \mathrm{H}$, $\left.\mathrm{CH}_{3}\right), 2.32\left(\mathrm{~s}, 3 \mathrm{H}, \mathrm{CH}_{3}\right), 6.82(\mathrm{~d}, 1 \mathrm{H}, \mathrm{Ar}-\mathrm{H}, J=7.5 \mathrm{~Hz})$, 7.14-7.16 (m, 2H, Ar-H), 7.36 (d, 1H, Ar-H, J=7.0 Hz), 7.44 (s, 1H, Ar-H), 7.52 (d, 1H, Ar-H, J=7.5 Hz), 7.68 (d, 1H, Ar-H, $J=7.0 \mathrm{~Hz}$ ), 11.11 (s, 1H, NH), 13.63 (s, 1H, NH); ${ }^{13} \mathrm{C}$ NMR (DMSO- $\left.d_{6}\right) \delta$ ppm: $21.0\left(\mathrm{CH}_{3}\right), 29.8\left(\mathrm{CH}_{3}\right), 111.3$, $115.4,118.1,121.4,124.6,127.2,131.8,132.5,134.6,137.4$, $140.7,146.3,152.4,157.8,162.5,164.1 ; \mathrm{MS}$ (ESI) $\mathrm{m} / \mathrm{z}: 334.0$ $[\mathrm{M}+\mathrm{H}]^{+}$; Anal. calcd. for $\mathrm{C}_{18} \mathrm{H}_{15} \mathrm{~N}_{5} \mathrm{O}_{2}$ (333.12): C, 64.86; H, 4.54; N, 21.01; found C, 64.69; H, 4.61; N, 21.12; HRMS 
(MALDI) calcd. for $\mathrm{C}_{18} \mathrm{H}_{15} \mathrm{~N}_{5} \mathrm{O}_{2}: 334.1304$, found: 350 . $334.1322[\mathrm{M}+\mathrm{H}]^{+}$.

\section{Pharmacological evaluation}

The details of the experimental protocols are provided in Supplementary materials.

\section{Metabolic investigations}

The study protocol was approved by the Research Ethics Committee at College of Pharmacy, King Saud University. Animals were maintained according to the guidelines of Animal Care Center, College of Pharmacy, King Saud University, and approved by the Local Animal Care and Use Committee of King Saud University. The details of the experimental protocols $^{40-42}$ are provided in Supplementary materials.

\section{Results and discussion Chemistry}

The synthetic pathway employed to prepare the target isatin derivatives is outlined in Scheme 1. The target compounds 6a-f, 7a-e, 8a-f and $\mathbf{9 a}-\mathbf{f}$ were obtained by the reaction of the appropriate indoline-2,3-diones $\mathbf{5 a}-\mathbf{f}$ with the hydrazinyl intermediates 1-4 in refluxed ethanol in the presence of a catalytic amount of glacial acetic acid with $70 \%-86 \%$ yields (Scheme 1).

IR spectra of the target compounds $6 \mathbf{a}-\mathbf{f}, 7 \mathbf{a}-\mathbf{e}, \mathbf{8 a}-\mathbf{f}$ and 9a-f showed absorption bands due to the $\mathrm{NH}$ groups in the region 3,347-3,421 $\mathrm{cm}^{-1}$, in addition to carbonyl bands in the region $1,697-1,718 \mathrm{~cm}^{-1}$. Their ${ }^{1} \mathrm{H}$ NMR spectra showed two singlet signals attributable to $\mathrm{NH}$ protons of the isatin and the hydrazine function $(=\mathrm{N}-\mathrm{NH}-)$ in the region

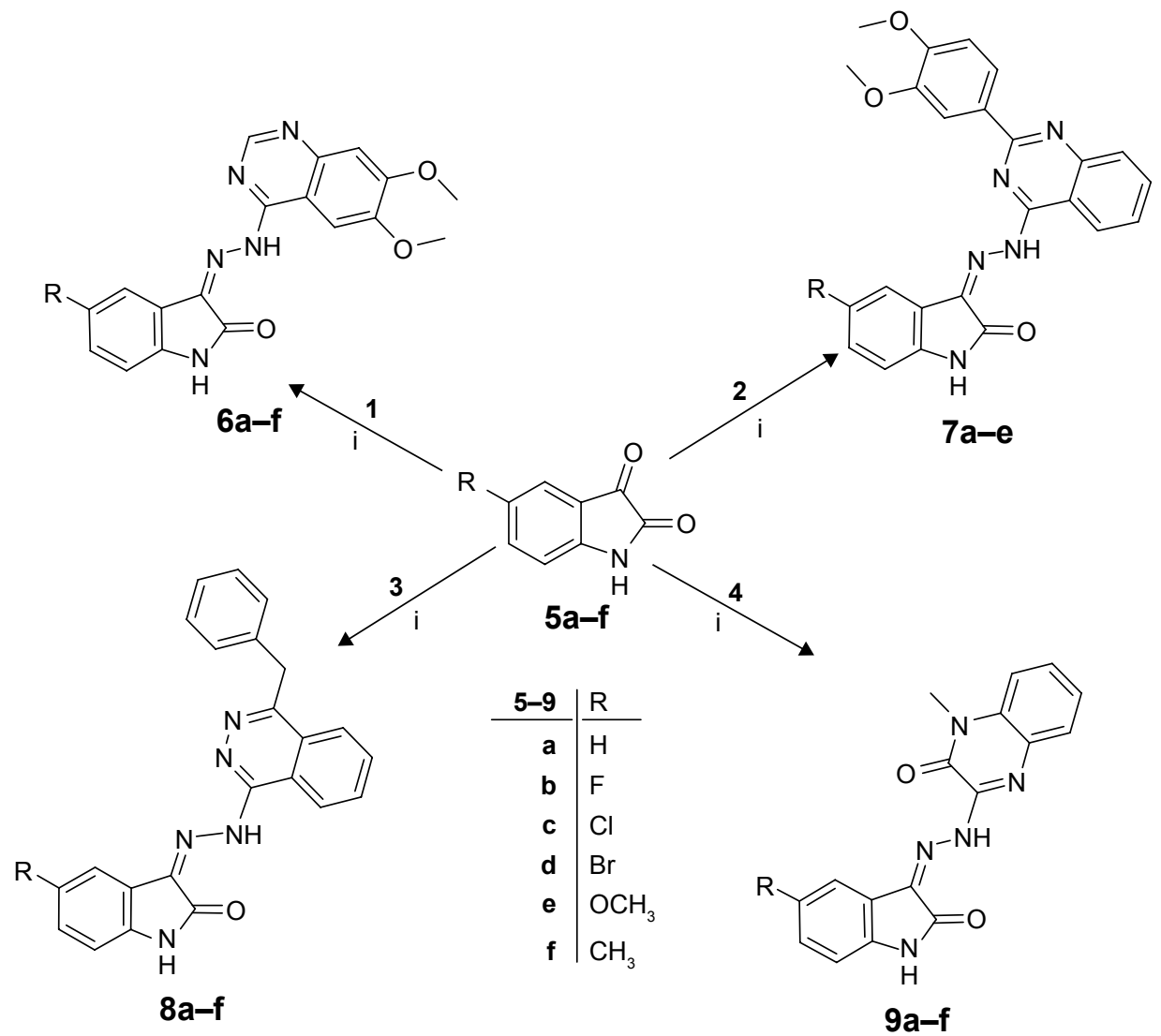

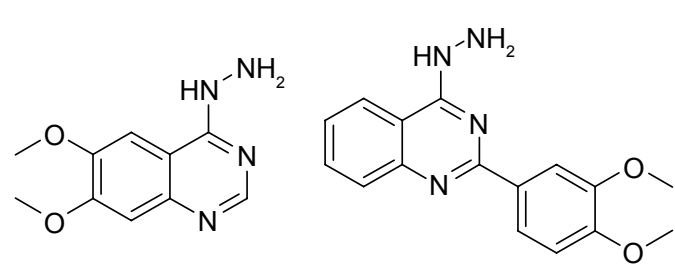

1
2<smiles>NNc1nnc(Cc2ccccc2)c2ccccc12</smiles>

3<smiles>Cn1c(=O)c(NN)nc2ccccc21</smiles>

4

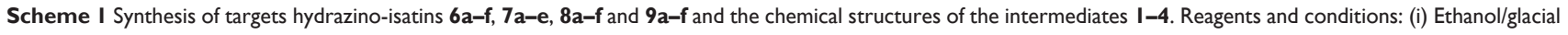
acetic acid (catalytic)/reflux for I h. 
$\delta 10.40-11.44$ and $11.24-13.81 \mathrm{ppm}$. Also, the methoxy $\left(-\mathrm{OCH}_{3}\right)$ protons of compounds $\mathbf{6 a}-\mathbf{f}$ appeared as singlet signals around $\delta 4.00 \mathrm{ppm}$, while the methoxy protons of derivatives 7a-e appeared around $\delta 3.80 \mathrm{ppm}$ in the ${ }^{1} \mathrm{H}$ NMR spectra. Furthermore, the $\left(-\mathrm{CH}_{2}\right)$ protons of benzylic moiety of 8a-f appeared as a singlet signal in the range $\delta 4.36-4.37$ ppm, while in case of $\mathbf{9 a}-\mathbf{f}$ the signals of the aliphatic protons $\left(\mathrm{N}-\mathrm{CH}_{3}\right)$ were observed as singlets near to $\delta 3.70 \mathrm{ppm}$.

\section{Pharmacological evaluation}

\section{Antiproliferative activity}

A total of 23 compounds were analyzed for cancer cell growth inhibitory activity. These studies were carried out using cells derived from human lung, colon and breast tumors (A-549, HT-29 and ZR-75 cells, respectively). This initial assessment of activity tested each compound in quadruplicate at a single concentration of $30 \mu \mathrm{M}$, if solubility permitted. As indicated in Table 1, compounds $\mathbf{8 b}-\mathbf{d}$ are the most potent congeners, inhibiting growth of all three cell lines with average growth inhibition values of $93.8,96.5$ and $96.4 \%$, respectively, at a test concentration of $30 \mu \mathrm{M}$. The rest of the compounds showed an average growth inhibition values from $6.1 \%-81.2 \%$ at the tested concentration levels.

Table I Antiproliferative (cell growth inhibitory activity at $30 \mu \mathrm{M}$ concentration) activity of the target compounds $\mathbf{6 a - f}, \mathbf{7 a - e , ~ 8 a - f}$ and $\mathbf{9 a - f}$ against HT-29, ZR-75 and A-549 cell lines

\begin{tabular}{lllll}
\hline Compound & HT-29 & ZR-75 & A-549 & $\begin{array}{l}\text { Average growth } \\
\text { inhibition \% }\end{array}$ \\
\hline 6a & $38.7 \pm 9.1$ & $50.4 \pm 19.4$ & $32.9 \pm 7.0$ & 40.7 \\
6b* & $13.2 \pm 10.5$ & $19.2 \pm 8.6$ & $7.1 \pm 8.2$ & 13.2 \\
6c & $5.5 \pm 3.2$ & $16.4 \pm 13.2$ & $10.9 \pm 11.1$ & 11.0 \\
6d* & $18.6 \pm 4.6$ & $4.2 \pm 12.7$ & $-4.3 \pm 16.4$ & 6.1 \\
6e* & $20.9 \pm 8.1$ & $32.0 \pm 18.6$ & $-10.4 \pm 8.5$ & 14.2 \\
6f & $49.3 \pm 5.5$ & $40.4 \pm 11.3$ & $20.5 \pm 15.0$ & 36.7 \\
7a & $38.1 \pm 6.8$ & $54.9 \pm 14.3$ & $58.6 \pm 10.5$ & 50.6 \\
7b & $9.6 \pm 9.2$ & $26.5 \pm 6.6$ & $4.9 \pm 8.5$ & 13.7 \\
7c & $5.3 \pm 7.9$ & $14.2 \pm 14.4$ & $10.9 \pm 12.6$ & 10.2 \\
7d* & $70.8 \pm 7.6$ & $78.8 \pm 7.6$ & $77.1 \pm 6.3$ & 75.6 \\
7e & $6.2 \pm 6.4$ & $23.1 \pm 8.2$ & $17.0 \pm 9.0$ & 15.4 \\
8a & $87.6 \pm 8.3$ & $54.3 \pm 9.4$ & $81.1 \pm 7.4$ & 74.4 \\
8b & $98.5 \pm 1.0$ & $86.3 \pm 5.4$ & $96.5 \pm 2.2$ & 93.8 \\
8c & $95.7 \pm 2.8$ & $96.1 \pm 2.1$ & $97.8 \pm 3.0$ & 96.5 \\
8d & $96.8 \pm 3.0$ & $97.2 \pm 4.2$ & $95.2 \pm 3.5$ & 96.4 \\
8e & $9.3 \pm 10.2$ & $30.9 \pm 18.1$ & $13.5 \pm 14.5$ & 17.9 \\
8f & $89.1 \pm 8.6$ & $69.6 \pm 6.6$ & $85.0 \pm 15.0$ & 81.2 \\
9a & $73.0 \pm 14.2$ & $52.1 \pm 11.9$ & $95.2 \pm 2.6$ & 73.4 \\
9b & $48.1 \pm 7.3$ & $22.6 \pm 14.2$ & $53.2 \pm 10.0$ & 41.3 \\
9c & $16.1 \pm 14.5$ & $29.8 \pm 14.3$ & $27.1 \pm 14.7$ & 24.3 \\
9d & $49.4 \pm 16.2$ & $62.1 \pm 18.5$ & $84.9 \pm 4.7$ & 65.5 \\
9e & $48.5 \pm 13.7$ & $76.0 \pm 2.8$ & $85.9 \pm 6.2$ & 70.2 \\
9f & $47.6 \pm 7.5$ & $71.0 \pm 10.8$ & $68.8 \pm 10.3$ & 62.5 \\
Sunitinib & $59.5 \pm 2.3$ & $90.7 \pm 4.5$ & $85.7 \pm 2.7$ & 78.7 \\
\hline & & & &
\end{tabular}

Note: *Tested concentration was $10 \mu \mathrm{M}$.
Table $2 \quad I_{50}$ of antiproliferative activity of the selected compounds $\mathbf{8 b}-\mathbf{d}$ and sunitinib against HT-29, ZR-75 and A-549 cell lines

\begin{tabular}{|c|c|c|c|c|}
\hline \multirow[t]{2}{*}{ Compound } & \multicolumn{3}{|l|}{$I_{50}(\mu M)$} & \multirow{2}{*}{$\begin{array}{l}\text { Average } \\
I C_{50}(\mu M)\end{array}$} \\
\hline & HT-29 & ZR-75 & A-549 & \\
\hline $8 b$ & $6.69 \pm 0.4$ & $13.25 \pm 2.0$ & $7.19 \pm 1.3$ & 9.04 \\
\hline $8 c$ & $5.31 \pm 1.2$ & $5.90 \pm 0.3$ & $5.39 \pm 0.6$ & 5.53 \\
\hline $8 d$ & $6.23 \pm 0.7$ & $7.77 \pm 2.7$ & $7.02 \pm 1.0$ & 7.01 \\
\hline Sunitinib & $10.14 \pm 0.8$ & $8.31 \pm 2.4$ & $5.87 \pm 0.3$ & 8.11 \\
\hline
\end{tabular}

Abbreviation: $\mathrm{IC}_{50}$, inhibitory concentration $50 \%$.

The most active promising compounds $\mathbf{8 b}-\mathbf{d}$ in the preliminary antiproliferative screening were subjected to quantitative inhibitory concentration $50 \%\left(\mathrm{IC}_{50}\right)$ determination for their cell growth inhibitory activity towards A-549, HT-29 and ZR-75 cancer cell lines and the results are presented in Table 2.

Compound $\mathbf{8 c}$ bearing 4-benzylphthalazine moiety exhibited the best average $\mathrm{IC}_{50}$ value of $5.53 \mu \mathrm{M}$, as compared with the positive control, sunitinib, which showed an average $\mathrm{IC}_{50}=8.11 \mu \mathrm{M}$. Therefore, compound $\mathbf{8 c}$ was subjected to deeper pharmacological investigations in order to gain insight into its pharmacological profile.

\section{Apoptosis and caspase $3 / 7$ activity}

Compound $8 \mathbf{c}$ was analyzed for apoptosis-inducing activity in cancer cells. These studies were carried out using cells derived from human lung (A-549). This further assessment of activity tested compounds in quadruplicate at concentrations equivalent to $\mathrm{IC}_{50}$ value to inhibit growth and a concentration 3-fold above the $\mathrm{IC}_{50}$ concentrations over a time course ranging from 2-48 h. As indicated in Figure 3, compound $\mathbf{8 c}$ at $5 \mu \mathrm{M}$ increased caspase activity by 3 -fold after $16 \mathrm{~h}$

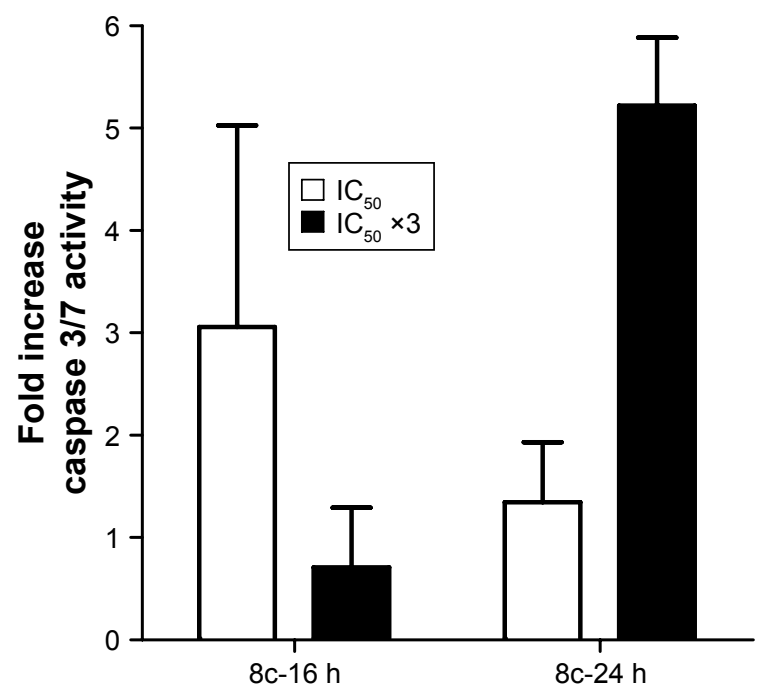

Figure 3 Caspase $3 / 7$ activity of compound $\mathbf{8 c}$. Abbreviation: $\mathrm{IC}_{50}$, inhibitory concentration $50 \%$. 

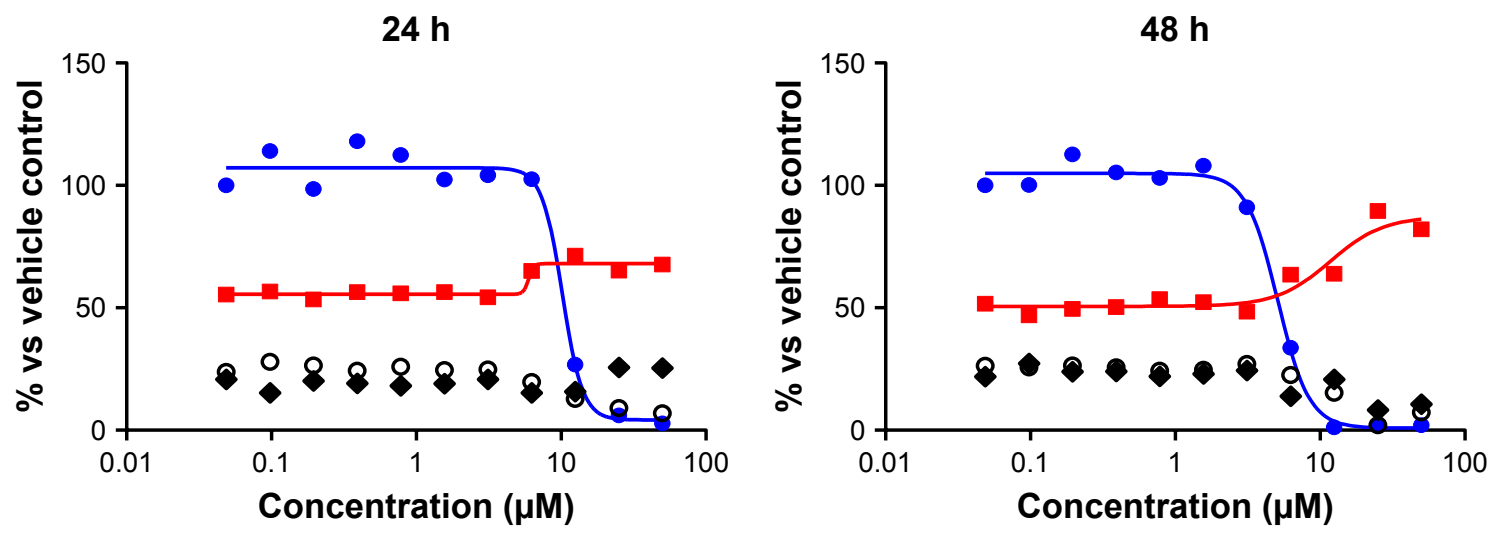

- Cell \# G1 $\bullet \mathrm{S} \quad \mathrm{O}$ G2/M

Figure 4 Cell cycle effects of compound $8 \mathrm{c}$ after 24 and $48 \mathrm{~h}$ of incubation.

of treatment and to over 5-fold after $24 \mathrm{~h}$ of treatment at a concentration of $15 \mu \mathrm{M}$.

\section{Cell cycle effects}

Compound $\mathbf{8 c}$ was analyzed for effects on various aspects of the cell cycle progression in human cancer cells. These studies were carried out using cells derived from lung adenocarcinoma (A-549). This follow-up assessment of activity tested compounds using immunofluorescent imaging of phosphorylated $\mathrm{Rb}$ protein and total DNA content of each cell to assess phase of cell cycle. The ability of test compounds to affect cell cycle distribution and $\mathrm{Rb}$ phosphorylation was tested over a range of concentrations less than $100 \mathrm{nM}$ to $50 \mu \mathrm{M}$. As shown in Figure 4, compound 8c produced dose-dependent effects on the tested parameters; however, compound $\mathbf{7 d}$ displayed no effects on the tested parameters (not shown). Compound $\mathbf{8 c}$ caused a significant reduction in the total cell number after $24 \mathrm{~h}$ of treatment with $\mathrm{IC}_{50}$ value $=10.19 \mu \mathrm{M}$ and with $\mathrm{IC}_{50}$ value $=5.11 \mu \mathrm{M}$ after $48 \mathrm{~h}$ (Table 3$)$.

In addition, compound $8 \mathrm{c}$ caused an increase in the percentage of cells in the G1 phase of the cell cycle with corresponding decrease in $\mathrm{S}$ and $\mathrm{G} 2 / \mathrm{M}$ phases. This suggests that part of the compound effects on growth may be attributable to the decreased rate of progression through the cell cycle and corresponding decrease in proliferation. By contrast, sunitinib caused a reduction in the percentage of cells in $\mathrm{G} 1$, with corresponding increases in S or G2/M phases. Arrest in G2 may represent a checkpoint blockade, whereas mitotic arrest may, in some cases, lead to mitotic catastrophe and subsequent programmed death of cells with multiple or aberrant nuclei.

As with other cell cycle parameters, levels of phosphorylated $\mathrm{Rb}$ protein were substantially reduced in a dosedependent manner by the control and the test compound $\mathbf{8 c}$. After $24 \mathrm{~h}$ of treatment, the $\mathrm{IC}_{50}$ value was lower than the $\mathrm{IC}_{50}$ value for reductions in the cell number caused by compound 8c (Table 3). This may support the hypothesis that inhibition of cyclin-dependent kinases by isatin compounds plays a role in their growth inhibitory activity. However, the correlation is less apparent at the 48 -h time point. Furthermore, compound $8 \mathbf{c}$ was analyzed for effects on total cellular levels of phosphorylated tyrosine residues in human cancer cells. These studies were carried out using cells derived from lung adenocarcinoma (A-549) and immunofluorescent imaging. The ability of the test compounds to affect acute serum stimulation of tyrosine phosphorylation was tested over a range of concentrations less than 100 to $50 \mu \mathrm{M}$. Compound $\mathbf{8 c}$ had no significant effect on P-Tyr labeling.

\section{Selectivity}

As an indicator of the selectivity for tumor cells, compound 8c was analyzed for its cell growth inhibitory activity in

Table $3 \mathrm{IC}_{50}$ for reductions in the total cell number and cell cycle effects of compound $\mathbf{8 c}$ and sunitinib

\begin{tabular}{|c|c|c|c|c|c|}
\hline \multirow[t]{2}{*}{ Compound } & \multicolumn{2}{|c|}{$\begin{array}{l}\mathrm{IC}_{50}(\mu \mathrm{M}) \text { for reductions } \\
\text { in the total cell number }\end{array}$} & \multicolumn{2}{|c|}{$\begin{array}{l}I_{50}(\mu \mathrm{M}) \text { for reduction } \\
\text { in } \mathbf{R b} \text { phosphorylation }\end{array}$} & \multirow[t]{2}{*}{ Cell cycle effects } \\
\hline & $24 \mathrm{~h}$ & $48 \mathrm{~h}$ & $24 \mathrm{~h}$ & $48 \mathrm{~h}$ & \\
\hline $8 c$ & $10.19 \pm 1.3$ & $5.1 I \pm I .1$ & $6.55 \pm 0.3$ & $6.18 \pm 0.1$ & GI increased, $S$ and $G 2 / M$ phase decrease \\
\hline Sunitinib & $12.54 \pm 2.9$ & $3.48 \pm 1.6$ & $3.16 \pm 0.1$ & $7.99 \pm 4.1$ & GI decreased and G2/M phase increased \\
\hline
\end{tabular}

Abbreviation: $\mathrm{IC}_{50}$, inhibitory concentration $50 \%$. 

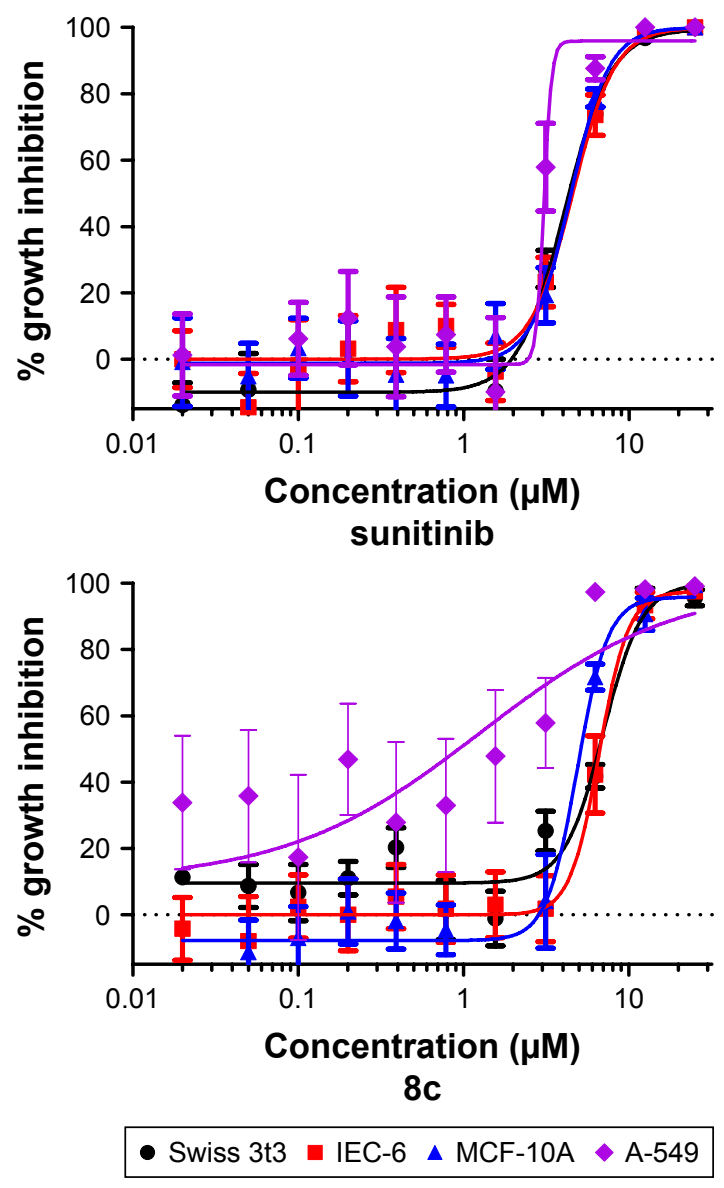

Figure $\mathbf{5}$ Selectivity profile of sunitinib and compound $\mathbf{8 c}$.

three non-tumorigenic cell lines. IEC-6 cells derived from rat intestine exhibit morphologic and karyotypic features of normal intestinal epithelial cells. ${ }^{43}$ Cultures derived from human fibrocystic mammary tissue (MCF-10A) are nontumorigenic and exhibit features of primary cultures of breast tissue including dome formation. ${ }^{44}$ Fibroblasts derived from embryonic tissue from mice (Swiss 3 t3 fibroblasts) are both non-tumorigenic and contact inhibited. ${ }^{45}$ For comparison, A-549 human non-small cell lung cancer (NSCLC) cell line was included. This assessment of growth inhibitory activity tested compounds in quadruplicate at maximum concentrations of $25 \mu \mathrm{M}$, followed by 10 serially diluted concentrations. As demonstrated in Figure 5 and Table 4, compound

Table 4 Selectivity for compound $\mathbf{8 c}$ and sunitinib toward tumor and non-tumorigenic cell lines

\begin{tabular}{|c|c|c|c|c|c|}
\hline \multirow[t]{2}{*}{ Compound } & \multicolumn{4}{|l|}{$I C_{50}(\mu M)$} & \multirow{2}{*}{$\begin{array}{l}\text { Mean } \\
\text { tumor } \\
\text { selectivity }\end{array}$} \\
\hline & $\begin{array}{l}\text { Intestine } \\
\text { IEC-6 }\end{array}$ & $\begin{array}{l}\text { Breast } \\
\text { MCF-I0A }\end{array}$ & $\begin{array}{l}\text { Fibroblast } \\
\text { Swiss 3t3 }\end{array}$ & $\begin{array}{l}\text { NSCLC } \\
\text { A-549 }\end{array}$ & \\
\hline $8 c$ & $6.60 \pm 1.1$ & $4.87 \pm 1.1$ & $6.98 \pm 0.6$ & $1.27 \pm 1.5$ & 4.8 \\
\hline Sunitinib & $4.56 \pm 0.9$ & $4.43 \pm 0.8$ & $4.07 \pm 0.5$ & $3.06 \pm 0.9$ & 1.4 \\
\hline
\end{tabular}

Abbreviations: $\mathrm{IC}_{50}$, inhibitory concentration $50 \%$; NSCLC, non-small cell lung cancer.
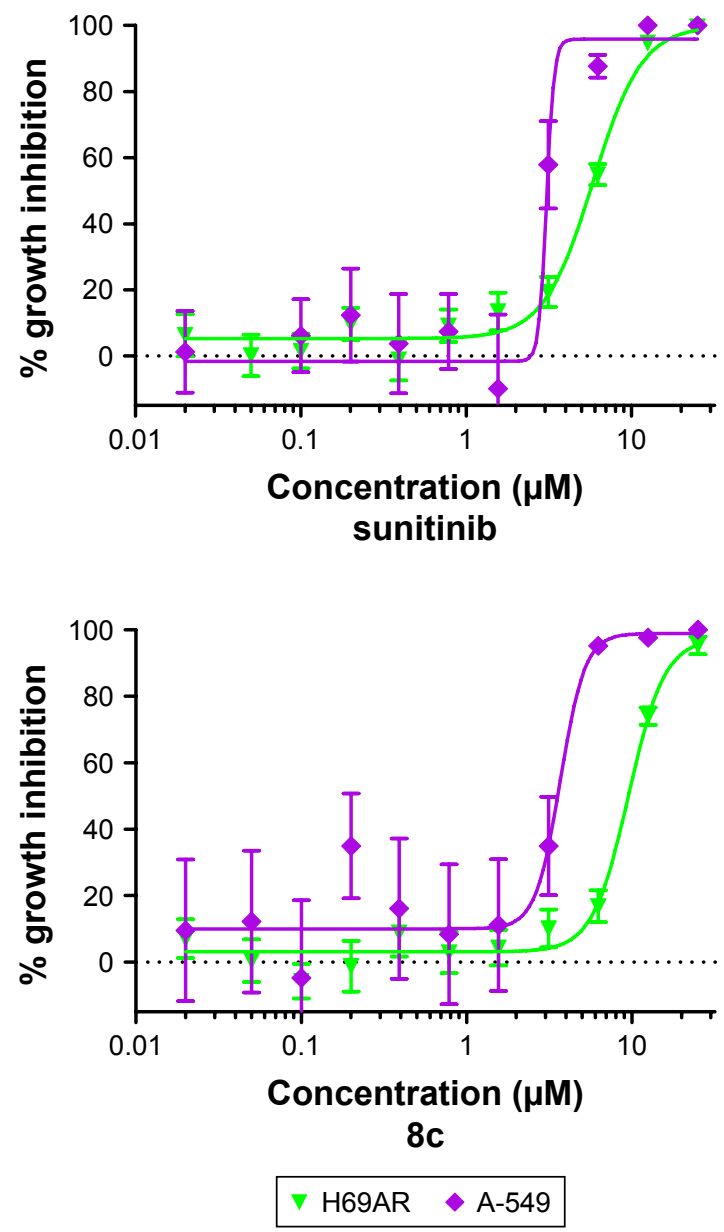

Figure 6 Activity of sunitinib and compound $8 \mathrm{c}$ against sensitive and resistant cancer cell lines.

8c inhibited growth in both normal and tumor cell lines by $>50 \%$. Compound $8 \mathrm{c}$ not only inhibited NSCLC with $\mathrm{IC}_{50}$ value $=1.27 \mu \mathrm{M}$ but also inhibited non-tumor cells less potently with 4.8 -fold selectivity value. For the control compound, sunitinib, there was a modest degree of selectivity (1.4-fold difference between mean $\mathrm{IC}_{50}$ in non-tumor cell lines versus the NSCLC cells).

\section{Multidrug-resistant lung cancer cell line}

Compound 8c was analyzed for cancer cell growth inhibitory activity in a sensitive NSCLC cell line (A-549) and a multidrug-resistant lung cancer cell line (NCI-H69AR) that expresses the $\mathrm{ABCC} 1$ efflux pump protein. This assessment of activity tested compound $8 \mathbf{c}$ in quadruplicate at maximum concentrations of $25 \mu \mathrm{M}$, followed by 10 serially diluted concentrations. As illustrated in Figure 6 and summarized in Table 5, compound $\mathbf{8 c}$ inhibited growth in both sensitive and resistant cancer cell lines with $\mathrm{IC}_{50}$ values $=1.3$ and $9.5 \mu \mathrm{M}$, respectively, being 7.5 -fold less sensitive toward the resistant 
Table 5 Cancer cell growth inhibitory activity of compound 8c and sunitinib toward sensitive (A-549) and resistant $\mathrm{NCl}-\mathrm{H} 69 \mathrm{AR}$ cancer cell lines

\begin{tabular}{|c|c|c|c|}
\hline \multirow[t]{2}{*}{ Compound } & \multicolumn{2}{|l|}{$I C_{50}(\mu M)$} & \multirow{2}{*}{$\begin{array}{l}\text { Fold } \\
\text { resistance }\end{array}$} \\
\hline & Sensitive A-549 & Resistant NCI-H69AR & \\
\hline $8 c$ & $1.3 \pm 1.5$ & $9.5 \pm 0.6$ & 7.5 \\
\hline Sunitinib & $3.1 \pm 0.9$ & $5.8 \pm 0.4$ & 1.9 \\
\hline
\end{tabular}

Abbreviation: $\mathrm{IC}_{50}$, inhibitory concentration $50 \%$.

NCI-H69AR cell line, indicating that this compound may be subjected to efflux by ABCC1. Sunitinib showed a lesser degree of fold resistance being 1.9-fold less sensitive toward the resistant NCI-H69AR cell line.

\section{Metabolic investigations}

The study of drug metabolism is a core part of the process of drug discovery and development; it has evolved from being a complementary step to that process to becoming crucial to it. ${ }^{46}$ Nowadays, metabolic profiles of new drugs have to be investigated prior to any clinical use of such drugs. This approach has been prejudiced by data accumulation assuming that poor pharmacokinetics is the main reason for failure of drug substances, in which the metabolic liability of a drug molecule is the primary determinant. ${ }^{47,48}$ In this study, comparison of the extracted ion chromatograms between incubations with or without RLMs as well as comparison of the product ion mass spectra of the postulated metabolites of $\mathbf{8 c}$ allowed the detection of ten metabolites. Such metabolites resulted from the incubation of $\mathbf{7 d}$ and 8c with RLMs that involved various metabolic reaction types, namely, demethylation for $\mathbf{7 d}$ and isomerization, reduction, hydroxylation and oxidation for $8 \mathbf{c}$ (Figure 7 and Scheme 2). Table 6 summarizes the product ions, retention times and metabolic reactions for the in vitro phase I $\mathbf{8 c}$ metabolites.

\section{Conclusion}

Quinazoline-isatin hybrids $\mathbf{6 a}-\mathbf{f}$ and $\mathbf{7 a - e}$, phthalazineisatin hybrids 8a-f and 1-methylquinoxaline-isatin hybrids 9a-f were synthesized and characterized with different spectroscopic techniques. The preliminary in vitro antiproliferative activity of the synthesized compounds against various human cancer cell lines revealed that compounds $\mathbf{8 b}-\mathbf{d}$ were the most active candidates. Therefore, they were subjected to quantitative $\mathrm{IC}_{50}$ determination. Detailed pharmacological investigations were carried out on the most promising compound $\mathbf{8 c}$ in order to gain insight into its pharmacological profile. Compound $\mathbf{8 c}$ induced apoptosis through increasing caspase $3 / 7$ activity by about 5 -fold at $15 \mu \mathrm{M}$ concentration using human cancer A-549 cell line. In addition, it displayed an increase in the G1 phase and a decrease in the $\mathrm{S}$ and $\mathrm{G} 2 / \mathrm{M}$ phases in the cell cycle effects assay and it showed $\mathrm{IC}_{50}$ value of $9.5 \mu \mathrm{M}$ against resistant NCI-H69AR cancer cell lines. In vitro metabolic profiling of compound $\mathbf{8 c}$ predicted its possible metabolites. Overall, the current study demonstrated that the new chemical entity $\mathbf{8 c}$ might be harnessed for cancer therapy after integration of the required preclinical studies.

\section{Supporting materials}

The details of the experimental methods that were adopted for the pharmacological investigations of the prepared compounds, the protocols that were used for metabolic studies and representative NMR $\left({ }^{1} \mathrm{H}\right.$ and $\left.{ }^{13} \mathrm{C}\right)$ spectra of the target compounds are provided as Supplementary materials.

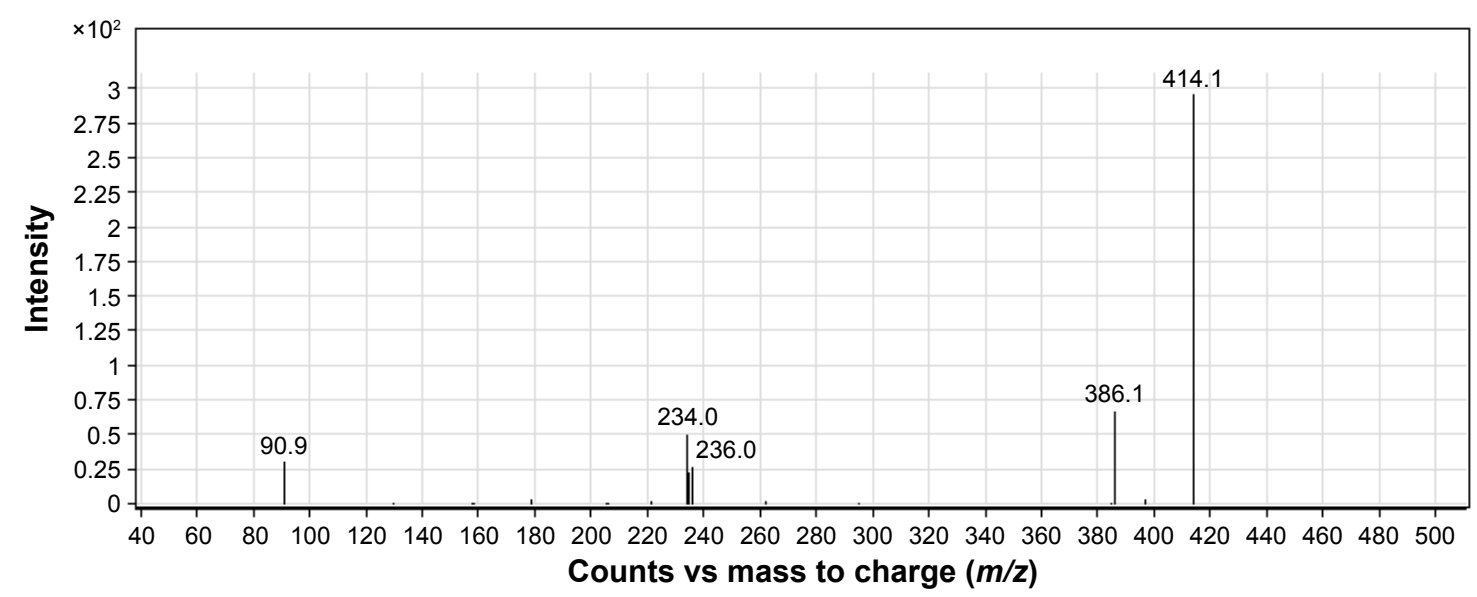

Figure $7 \mathrm{~A}$ representative product ion spectrum of compound $\mathbf{8 c}$ (retention time $=36.40 \mathrm{~min}$ ) 


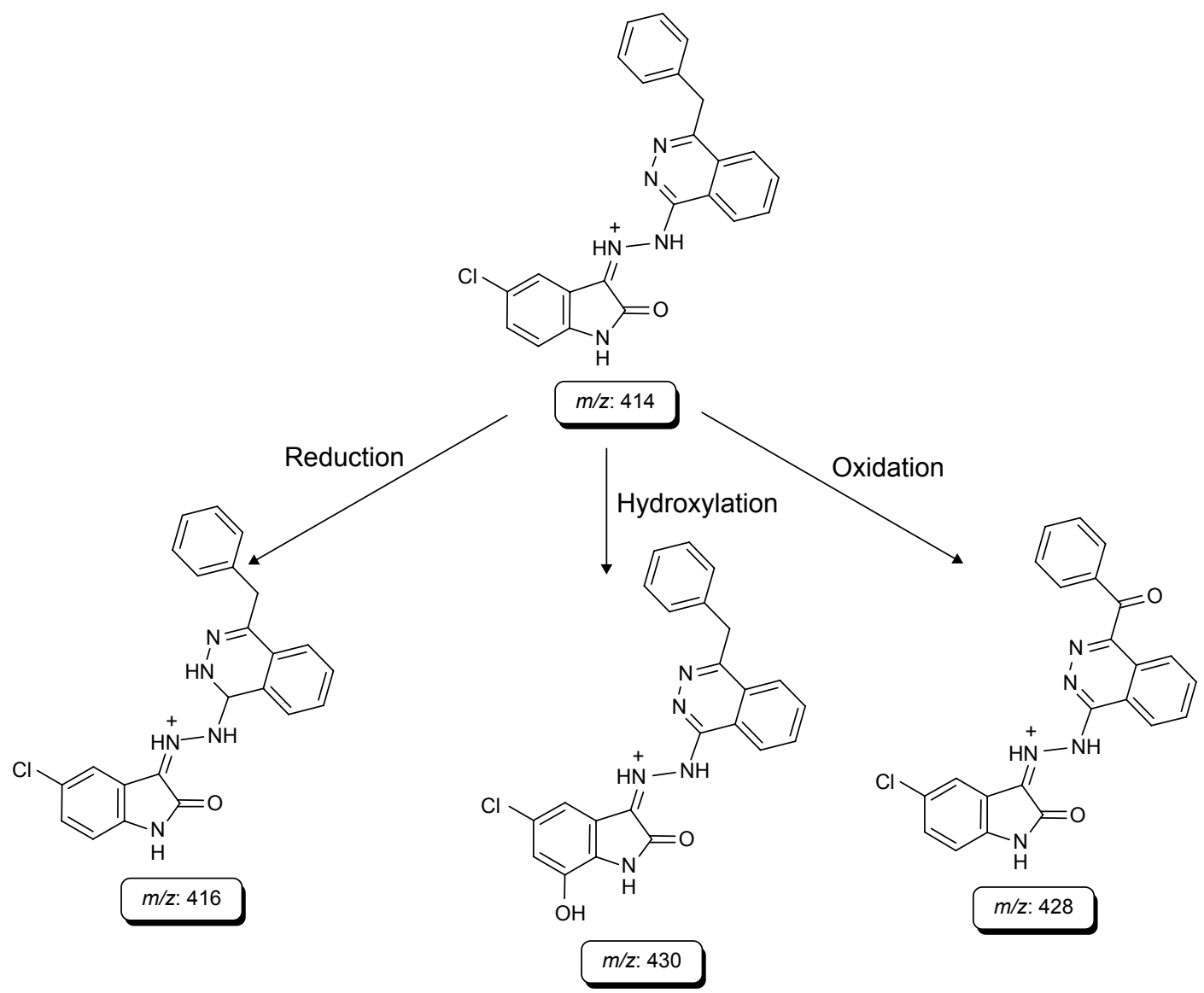

Scheme 2 Postulated in vitro metabolic pathway of compound $8 \mathrm{c}$.

Table 6 In vitro RLMs metabolites of compound 8c

\begin{tabular}{|c|c|c|c|c|c|}
\hline Compound & Compound/metabolite & Metabolic reaction & $\begin{array}{l}\text { Monoisotopic } \\
\text { mass }(\mathrm{m} / \mathrm{z})\end{array}$ & $\begin{array}{l}\text { Product } \\
\text { ion }(\mathrm{m} / \mathrm{z})\end{array}$ & $\begin{array}{l}\text { Retention } \\
\text { time (min) }\end{array}$ \\
\hline \multirow[t]{11}{*}{$8 c$} & $8 c$ & - & 414 & $386,236,234,91$ & 36.40 \\
\hline & 8c Reduced & Reduction & 416 & $388,234,91$ & 36.35 \\
\hline & 8c Reduced isomer & Reduction and isomerization & 416 & $388,234,91$ & 43.82 \\
\hline & 8c Hydroxylated & Arom hydroxylation & 430 & 234,181 & 31.62 \\
\hline & 8c Hydroxylated isomer I & Arom hydroxylation and isomerization & 430 & $412,233,107$ & 32.30 \\
\hline & 8c Hydroxylated isomer 2 & Arom hydroxylation and isomerization & 430 & $412,250,233,107$ & 38.96 \\
\hline & 8c Hydroxylated isomer 3 & Arom hydroxylation and isomerization & 430 & $412,233,107$ & 40.34 \\
\hline & 8c Oxygenated & oxidation & 428 & $322,107,79$ & 27.25 \\
\hline & 8c Oxygenated isomer I & oxidation and isomerization & 428 & $322,107,79$ & 25.70 \\
\hline & 8c Oxygenated isomer 2 & oxidation and isomerization & 428 & 105,77 & 40.35 \\
\hline & 8c Oxygenated isomer 3 & oxidation and isomerization & 428 & $179,105,77$ & 44.24 \\
\hline
\end{tabular}

Abbreviations: RLM, rat liver microsome; Arom, aromatic.

\section{Acknowledgment}

This project was funded by the National Plan for Science, Technology and Innovation (MAARIFAH), King Abdulaziz City for Science and Technology, Kingdom of Saudi Arabia, Award Number 11-MED1924-02.

\section{Disclosure}

The authors report no conflicts of interest in this work.

\section{References}

1. Bérubé G. An overview of molecular hybrids in drug discovery. Expert Opin Drug Discov. 2016;11(3):281-305.

2. Fortin S, Bérubé G. Advances in the development of hybrid anticancer drugs. Expert Opin Drug Discov. 2013;8(8):1029-1047.

3. Viegas-Junior C, Danuello A, da Silva Bolzani V, Barreiro EJ, Fraga CA. Molecular hybridization: a useful tool in the design of new drug prototypes. Curr Med Chem. 2007;14(17):1829-1852.

4. Meunier B. Hybrid molecules with a dual mode of action: dream or reality? Acc Chem Res. 2008;41(1):69-77. 
5. Glover V, Halket JM, Watkins PJ, Clow A, Goodwin BL, Sandler M. Isatin: identity with the purified endogenous monoamine oxidase inhibitor tribulin. J Neurochem. 1988;51(2):656-659.

6. Vine KL, Matesic L, Locke JM, Ranson M, Skropeta D. Cytotoxic and anticancer activities of isatin and its derivatives: a comprehensive review from 2000-2008. Anticancer Agents Med Chem. 2009;9(4): 397-414.

7. Sun L, Liang C, Shirazian S, et al. Discovery of 5-[5-fluoro-2-oxo1,2-dihydroindol-(3Z)-ylidenemethyl]-2,4-dimethyl-1 $H$-pyrrole-3carboxylic acid (2-diethylaminoethyl)amide, a novel tyrosine kinase inhibitor targeting vascular endothelial and platelet-derived growth factor receptor tyrosine kinase. J Med Chem. 2003;46(7):1116-1119.

8. Prenen H, Cools J, Mentens N, et al. Efficacy of the kinase inhibitor SU11248 against gastrointestinal stromal tumor mutants refractory to imatinib mesylate. Clin Cancer Res. 2006;12(8):2622-2627.

9. Motzer RJ, Michaelson MD, Redman BG, et al. Activity of SU11248, a multitargeted inhibitor of vascular endothelial growth factor receptor and platelet-derived growth factor receptor, in patients with metastatic renal cell carcinoma. J Clin Oncol. 2006;24(1):16-24.

10. McCormack PL. Nintedanib: first global approval. Drugs. 2015;75(1): 129-139.

11. Roth GJ, Heckel A, Colbatzky F, et al. Design, synthesis, and evaluation of indolinones as triple angiokinase inhibitors and the discovery of a highly specific 6-methoxycarbonyl-substituted indolinone (BIBF 1120). J Med Chem. 2009;52(14):4466-4480.

12. Krug M, Hilgeroth A. Recent advances in the development of multikinase inhibitors. Mini Rev Med Chem. 2008;8(13):1312-1327.

13. Ibrahim HS, Abou-Seri SM, Abdel-Aziz HA. 3-Hydrazinoindolin-2-one derivatives: chemical classification and investigation of their targets as anticancer agents. Eur J Med Chem. 2016;122:366-381.

14. Abdel-Aziz HA, Elsaman T, Al-Dhfyan A, Attia MI, Al-Rashood KA, Al-Obaid AR. Synthesis and anticancer potential of certain novel 2-oxo- $N^{\prime}$-(2-oxoindolin-3-ylidene)- $2 \mathrm{H}$-chromene-3-carbohydrazides. Eur J Med Chem. 2013;70:358-363.

15. Eldehna WM, Altoukhy A, Mahrous H, Abdel-Aziz HA. Design, synthesis and QSAR study of certain isatin-pyridine hybrids as potential anti-proliferative agents. Eur J Med Chem. 2015;90:684-694.

16. Hassan TA, Kadi AA, Abdel-Aziz HA. Novel $N, N^{\prime}$-hydrazino-bis-isatin derivatives with selective activity against multidrug-resistant cancer cells. United States Patent US US20120252860. 2012

17. Ibrahim HS, Abou-seri SM, Ismail NS, Elaasser MM, Aly MH, AbdelAziz HA. Bis-isatin hydrazones with novel linkers: synthesis and biological evaluation as cytotoxic agents. Eur J Med Chem. 2016;108: 415-422.

18. Gudipati R, Anreddy RN, Manda S. Synthesis, anticancer and antioxidant activities of some novel $N$-(benzo[d]oxazol-2-yl)-2-(7-or 5substituted-2-oxoindolin-3-ylidene)-hydrazinecarboxamide derivatives. J Enzyme Inhib Med Chem. 2011;26(6):813-818.

19. Taher AT, Khalil NA, Ahmed EM. Synthesis of novel isatin-thiazoline and isatin-benzimidazole conjugates as anti-breast cancer agents. Arch Pharm Res. 2011;34(10):1615-1621.

20. Solomon VR, Hu C, Lee H. Hybrid pharmacophore design and synthesis of isatin-benzothiazole analogs for their anti-breast cancer activity. Bioorg Med Chem. 2009;17(21):7585-7592.

21. Havrylyuk D, Kovach N, Zimenkovsky B, Vasylenko O, Lesyk R. Synthesis and anticancer activity of isatin-based pyrazolines and thiazolidines conjugates. Arch Pharm (Weinheim). 2011;344(8):514-522.

22. Ramshid PK, Jagadeeshan S, Krishnan A, Mathew M, Nair SA, Pillai MR. Synthesis and in vitro evaluation of some isatin-thiazolidinone hybrid analogues as anti-proliferative agents. Med Chem. 2010;6(5): 306-312.

23. Kaminskyy D, Khyluk D, Vasylenko O, Zaprutko L, Lesky R. A facile synthesis and anticancer activity evaluation of spiro [thiazolidinoneisatin] conjugates. Sci Pharm. 2011;79(4):763-777.

24. Havrylyuk D, Zimenkovsky B, Vasylenko O, Gzella A, Lesyk R. Synthesis of new 4-thiazolidinone-, pyrazoline-, and isatin-based conjugates with promising antitumor activity. J Med Chem. 2012;55(20): 8630-8641.
25. Wang S, Zhao Y, Zhang G, Lv Y, Zhang N, Gong P. Design, synthesis and biological evaluation of novel 4-thiazolidinones containing indolin2-one moiety as potential antitumor agent. Eur J Med Chem. 2011;46(8): 3509-3518.

26. Solomon VR, Hu C, Lee H. Design and synthesis of anti-breast cancer agents from 4-piperazinylquinoline: a hybrid pharmacophore approach. Bioorg Med Chem. 2010;18(4):1563-1572.

27. Sharma M, Sharma S, Buddhiraja A, Saxena AK, Nepali K, Bedi PMS. Synthesis and cytotoxicity studies of 3, 5-diaryl $N$-acetyl pyrazolineisatin hybrids. Med Chem Res. 2014;23(10):4337-4344.

28. Cohen MH, Johnson JR, Chen YF, Sridhara R, Pazdur R. FDA drug approval summary: erlotinib (Tarceva) tablets. Oncologist. 2005;10(7): $461-466$.

29. Thornton K, Kim G, Maher VE, et al. Vandetanib for the treatment of symptomatic or progressive medullary thyroid cancer in patients with unresectable locally advanced or metastatic disease: US Food and Drug Administration drug approval summary. Clin Cancer Res. 2012; 18(14):3722-3730.

30. Bold G, Altmann KH, Frei J, et al. New anilinophthalazines as potent and orally well absorbed inhibitors of the VEGF receptor tyrosine kinases useful as antagonists of tumor-driven angiogenesis. $J$ Med Chem. 2000;43(12):2310-2323.

31. Dumas J, Dixon JA. VEGF receptor kinase inhibitors: phthalazines, anthranilamides and related structures. Expert Opin Ther Pat. 2005; 15(6):647-658.

32. Deeks ED. Olaparib: first global approval. Drugs. 2015;75(2):231-240.

33. Pinheiro AC, Mendonça Nogueira TC, de Souza MV. Quinoxaline nucleus: a promising scaffold in anti-cancer drug discovery. Anticancer Agents Med Chem. 2016;16(10):1339-1352.

34. Wu WY, Cao SL, Mao BB, et al. Synthesis and antiproliferative evaluation of hybrids of indolin-2-one and quinazoline-4-(3H)-one linked via imine bond. Lett Drug Des Discov. 2013;10(1):61-66.

35. Fares M, Eldehna WM, Abou-Seri SM, Abdel-Aziz HA, Aly MH, Tolba MF. Design, synthesis and in vitro antiproliferative activity of novel isatin-quinazoline hybrids. Arch Pharm (Weinheim). 2015;348(2): 144-154.

36. Eldehna WM, Fares M, Ceruso M, et al. Amido/ureido substituted benzenesulfonamides-isatin conjugates as low nanomolar/subnanomolar inhibitors of the tumor-associated carbonic anhydrase isoform XII. Eur J Med Chem. 2016;110:259-266.

37. He J, Wang X, Zhao X, Liang Y, He H, Fu L. Synthesis and antitumor activity of novel quinazoline derivatives containing thiosemicarbazide moiety. Eur J Med Chem. 2012;54:925-930.

38. El-Feky S, Bayoumy BE. Cyclocondensation of 4-benzyl-1hydrazinophthalazine. J Prakt Chem. 1990;332(6):1041-1048.

39. Makino K, Sakata G, Morimoto K, Ochiai Y. A facile synthesis of novel tricyclic compounds, tetrazoloquinoxalines and 1,2, 4-triazoloquinoxalines. Heterocycles. 1985;23(8):2025-2034.

40. Gill HJ, Tingle MD, Park BK. $N$-Hydroxylation of dapsone by multiple enzymes of cytochrome P450: implications for inhibition of haemotoxicity. Br J Clin Pharmacol. 1995;40(6):531-538.

41. Lowry OH, Rosebrough NJ, Farr AL, Randall RJ. Protein measurement with the Folin phenol reagent. J Biol Chem. 1951;193(1): $265-275$.

42. Billings RE. Sex differences in rats in the metabolism of phenytoin to 5-(3, 4-dihydroxyphenyl)-5-phenylhydantoin. J Pharmacol Exp Ther. 1983;225(3):630-636.

43. Quaroni A, Wands J, Trelstad RL, Isselbacher KJ. Epithelioid cell cultures from rat small intestine. Characterization by morphologic and immunologic criteria. J Cell Biol. 1979;80(2):248-265.

44. Soule HD, Maloney TM, Wolman SR, et al. Isolation and characterization of a spontaneously immortalized human breast epithelial cell line, MCF-10. Cancer Res. 1990;50(18):6075-6086.

45. Todaro GJ, Green H. Quantitative studies of the growth of mouse embryo cells in culture and their development into established lines. J Cell Biol. 1963;17:299-313.

46. Kumar GN, Surapaneni S. Role of drug metabolism in drug discovery and development. Med Res Rev. 2001;21(5):397-411. 
47. Caldwell GW. Compound optimization in early- and late-phase drug discovery: acceptable pharmacokinetic properties utilizing combined physicochemical, in vitro and in vivo screens. Curr Opin Drug Discov Devel. 2000;3(1):30-41.
48. Kennedy T. Managing the drug discovery/development interface. Drug Discov Today. 1997;2(10):436-444.

\section{Publish your work in this journal}

Drug Design, Development and Therapy is an international, peerreviewed open-access journal that spans the spectrum of drug design and development through to clinical applications. Clinical outcomes, patient safety, and programs for the development and effective, safe, and sustained use of medicines are the features of the journal, which has also been accepted for indexing on PubMed Central. The manuscript management system is completely online and includes a very quick and fair peer-review system, which is all easy to use. Visit http://www.dovepress.com/testimonials.php to read real quotes from published authors.

Submit your manuscript here: http://www.dovepress.com/drug-design-development-and-therapy-journal 